\title{
Neuroprotective Potential of Chrysin: Mechanistic Insights and Therapeutic Potential for Neurological Disorders
}

\author{
Awanish Mishra ${ }^{1,2, *}$, Pragya Shakti Mishra ${ }^{3}$, Ritam Bandopadhyay ${ }^{2}$, Navneet Khurana ${ }^{2}$ (D), \\ Efthalia Angelopoulou ${ }^{4}$, , Yam Nath Paudel ${ }^{5}\left(\mathbb{D}\right.$ and Christina Piperi ${ }^{4}(\mathbb{D}$ \\ 1 Department of Pharmacology and Toxicology, National Institute of Pharmaceutical Education and \\ Research (NIPER)—Guwahati, Changsari, Kamrup 781101, Assam, India \\ 2 Department of Pharmacology, School of Pharmaceutical Sciences, Lovely Professional University, \\ Phagwara 144411, Punjab, India; ritambanerjee02@gmail.com (R.B.); navneet.18252@lpu.co.in (N.K.) \\ 3 Department of Nuclear Medicine, Sanjay Gandhi Post Graduate Institute of Medical Sciences (SGPGIMS), \\ Lucknow 226014, Uttar Pradesh, India; pragya.mishra640@gmail.com \\ 4 Department of Biological Chemistry, Medical School, National and Kapodistrian University of Athens, \\ 11527 Athens, Greece; angelthal@med.uoa.gr (E.A.); cpiperi@med.uoa.gr (C.P.) \\ 5 Neuropharmacology Research Strength, Jeffrey Cheah School of Medicine and Health Sciences, \\ Monash University Malaysia, Bandar Sunway 47500, Selangor, Malaysia; yam.paudel@monash.edu \\ * Correspondence: awanish1985@gmail.com; Tel.: +91-972-155-4158 or +91-829-976-4600
}

\section{check for} updates

Citation: Mishra, A.; Mishra, P.S.; Bandopadhyay, R.; Khurana, N.; Angelopoulou, E.; Paudel, Y.N.; Piperi, C. Neuroprotective Potential of Chrysin: Mechanistic Insights and Therapeutic Potential for Neurological Disorders. Molecules 2021, 26, 6456. https://doi.org/ $10.3390 /$ molecules 26216456

Academic Editor: Maja Jazvinšćak Jembrek

Received: 3 August 2021

Accepted: 21 October 2021

Published: 26 October 2021

Publisher's Note: MDPI stays neutral with regard to jurisdictional claims in published maps and institutional affiliations.

Copyright: (c) 2021 by the authors. Licensee MDPI, Basel, Switzerland. This article is an open access article distributed under the terms and conditions of the Creative Commons Attribution (CC BY) license (https:// creativecommons.org/licenses/by/ $4.0 /)$.

\begin{abstract}
Chrysin, a herbal bioactive molecule, exerts a plethora of pharmacological effects, including anti-oxidant, anti-inflammatory, neuroprotective, and anti-cancer. A growing body of evidence has highlighted the emerging role of chrysin in a variety of neurological disorders, including Alzheimer's and Parkinson's disease, epilepsy, multiple sclerosis, ischemic stroke, traumatic brain injury, and brain tumors. Based on the results of recent pre-clinical studies and evidence from studies in humans, this review is focused on the molecular mechanisms underlying the neuroprotective effects of chrysin in different neurological diseases. In addition, the potential challenges, and opportunities of chrysin's inclusion in the neurotherapeutics repertoire are critically discussed.
\end{abstract}

Keywords: chrysin; antioxidant; neuroprotective agents; neurological disorders; epilepsy; neurodegenerative diseases

\section{Introduction}

The Global Burden of Disease (GBD) study and the World Health Organization (WHO) resources report that the overall health effects of neurological disorders have been underestimated. With a constant increase in the global age of the population, the augmented global burden of neurological disorders is posing a significant challenge to the maintenance of health care systems in developing and developed countries. Limited information is available pertaining to the prevalence, incidence and disease burden related to neurological disorders in India [1]. Over the past decade, the augmented incidence of neurological disorders has adversely affected quality of life, with severe socioeconomic consequences. Due to the elevated contribution of injury-related and non-communicable neurological disorders, research has been focused on the development of suitable management strategies. The most common neurological disorders include stroke, epilepsy, Alzheimer's disease (AD), Parkinson's disease (PD), multiple sclerosis (MS), cerebral palsy, brain tumor, and traumatic brain injury (TBI), being responsible for major disabilities worldwide [1-5].

Most of the currently available pharmacotherapeutic approaches provide merely symptomatic relief. Moreover, drug-associated adverse effects often complicate management, and further worsen the quality of life of these patients. Therefore, in the last decade, most of the research work has been focused on finding suitable alternatives with better safety profiles. In this regard, plant-derived functional foods with a wide variety of therapeutic and safety properties have gained growing attention among researchers. In general, 
the secondary metabolites of plants, including alkaloids, flavonoids, saponins, terpenes, etc., carry therapeutic potential. Flavonoids are bioactive molecules, derived from various plants and animal sources. Thousands of flavonoids have been reported carrying a broad spectrum of health benefits $[3,4,6]$.

In the human diet, flavonoids represent the biggest group of plant-derived polyphenolic substances. On average, dietary flavonoid consumption is about $50-800 \mathrm{mg} /$ day. As has been already extensively reviewed elsewhere, flavonoids exert a beneficial role on health due to their anti-oxidant, anti-inflammatory, antiviral and anti-carcinogenic properties via several cellular signaling pathways [7]. Flavonoid-rich foods, such as green tea, cocoa, and blueberry, exert beneficial effects via the interactions of flavonoids with several molecular targets. For instance, epigallocatechin gallate (EGCG), sequestered in red wine, chocolate and green tea, has been demonstrated to inhibit $A \beta$-induced neuronal apoptosis and caspase activity, promoting the survival of neurons in the hippocampus [8]. In addition, a blackberry-supplemented diet, which is enriched in polyphenols, has been associated with improved motor and cognitive performance in aged rat models [9]. Among family members, chrysin appears as a promising natural flavonoid, exhibiting an array of neuroprotective effects by attenuating oxidative stress, neuroinflammation, and apoptosis [3,6,7]. Chrysin, also known as chrysinic acid, belongs to the class of flavones. It is mainly obtained from honey, propolis, fruits and vegetables, primarily from the plants Yerba Santa, Pelargonium crispum, Passiflora incarnate, marsh skullcap and Oroxylem indicum. It possesses various pharmacological properties, including anti-inflammatory, anti-tumor, anti-asthmatic, antihyperlipidemic, cardioprotective, neuroprotective and renoprotective $[3,8]$.

Although there are several reviews on the roles of flavonoids in health and disease, herein, we mainly address the neuroprotective effects of chrysin, specifically in neurological disorders, based on the accumulating pre-clinical evidence, and discuss its emerging therapeutic potential as well limitations that need to be overcome for its effective clinical use.

\section{Chemistry and Pharmacokinetics of Chrysin}

Chrysin consists of two fused rings (A and C) attached with a phenyl ring (B) at the second position of the $C$ ring. In addition, at positions 5 and 7 of ring $A$, a hydroxyl group is attached (Figure 1) [3]. Polyphenols are not absorbed easily, especially in the form of esters, glycosides, and polymers. Due to their low absorption and high rate of metabolism and elimination, they possess poor intrinsic activity. Polyphenols degrade into aglycones and various aromatic acids after their hydrolyzation by intestinal enzymes. Aglycones are cardiac glycosides, considered as the most potent glycosides. Naturally occurring flavonoids get metabolized by phase I and phase II reactions (conjugation with methylation, sulfation and glucuronidation) and are eliminated from the body.

To address the pharmacological benefits and bioavailability of chrysin, it is necessary to understand the role of efflux transporters and the fate of its metabolites. There are three main transporters for chrysin conjugates: (a) the multidrug resistance-associated protein (MRP2), (b) the breast cancer resistance protein (BCRP), and (c) the ATP binding cassette (ABC). MRP2, also known as ABCC2, is a withdrawal efflux transporter that delivers anions, including drug conjugates and conjugated bilirubin. It is mainly expressed in the liver, kidney, and placenta. The chrysin metabolites are transported in Caco-2 cells through MRP2 [10]. These conjugates may be hydrolyzed by sulfatases and glucuronides to chrysin after their efflux into the small intestine. Studies using Caco-2 cell lines have shown that chrysin possesses favorable membrane transport properties [10]. However, a large amount of unchanged chrysin in stool samples indicates its poor intestinal absorption. BCRP (also known as ABCG2), an important efflux transporter of the $\mathrm{ABC}$ family of proteins for phase 2 metabolites (chrysin conjugates), are situated at the apical membrane of enterocytes and hepatocytes.

The anion inhibitor, MK-571, has been reported to reduce the elimination of chrysin metabolites (glucuronide and sulphate conjugates) in Caco-2 cells, suggesting that MRP2 
may inhibit the efflux of chrysin glucuronide and sulfate conjugates up to $71 \%$ [11]. The lethal dose of chrysin through the oral route is $4350 \mathrm{mg} / \mathrm{kg}$ [12].<smiles>O=c1cc(-c2ccccc2)oc2cc(O)cc(O)c12</smiles>

\section{Chrysin}

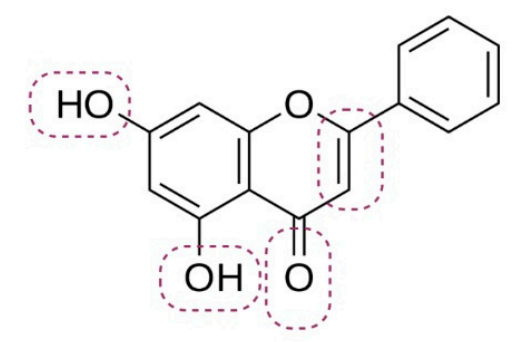

Anti-inflammatory Activity<smiles>O=c1cc(-c2ccccc2)oc2cc(O)cc(O)c12</smiles>

Antioxidant Activity

Figure 1. Chemical structure of chrysin and important pharmacophores for anti-inflammatory and anti-oxidant activity.

The major limitation of chrysin is its poor bioavailability, mainly due to its high metabolism. It is extensively metabolized by the intestine, liver, and several target cells, via conjugation, biotransformation, and the production of glucuronides and sulfate derivatives. Chrysin displays a very low distribution volume, and its oral bioavailability is about $0.003-0.02 \%$. The urine and plasma levels of chrysin metabolites-sulfonate and glucuronide-are very low, while bile contains the highest concentrations [13]. However, significant efforts are currently being made towards overcoming this limitation, and are discussed below.

\section{Potential Neuroprotective Mechanisms of Chrysin}

Chrysin has been reported to exert neuroprotective effects through different mechanisms, including anti-oxidant, anti-inflammatory and anti-apoptotic functions, MAO inhibition and GABA mimetic properties. The neuroprotective mechanisms of chrysin are illustrated in Figures 2 and 3.

\subsection{Chrysin as an Anti-Oxidant Agent}

Chrysin is a flavonoid, possessing a diphenylpropane (C6C3C6) skeleton system. In the structure-activity relationship studies, it has been shown that the diphenylpropane (C6C3C6) skeleton and the position of hydroxyl (-OH) substituents are very important for chrysin's anti-oxidant and anti-inflammatory activities (Figure 1). The further substitution of these hydroxyl groups with methoxy or ethoxy groups causes reductions in the antioxidant and anti-inflammatory activities of chrysin, while $\mathrm{C}=\mathrm{C}$ (between positions 2 and 3 ) 
is also important for these activities. The important pharmacophores of chrysin and the corresponding biological activities are illustrated in Figure 1 [14].

The Nuclear factor erythroid 2-related factor 2 (Nrf2), an important transcription factor for mediating the anti-oxidant effects, is upregulated by chrysin [15]. Upon activation, Nrf2 uncouples from Keap1 and migrates to the nucleus, where it binds to the anti-oxidant response element (ARE) and activates the downstream processing of heme oxygenase-1 (HO-1) and $\mathrm{NAD}(\mathrm{P}) \mathrm{H}$ quinone oxidoreductase 1 (NQO-1) (Figure 3). The downstream signaling of Nrf2 stimulates the production of anti-oxidant factors (SOD, GSH, and GST), and thus prevents oxidative stress-induced cellular damage [15].

The principal mediators of oxidative stress involve various types of reactive oxygen species (ROS) [16]. During oxidative stress, the fine balance between ROS production and removal is disrupted, leading to the accumulation of ROS inside the cell $[16,17]$. The increased ROS expression inside the cell leads to neurodegeneration via increased lipid peroxidation, mitochondrial dysfunction, and the activation of apoptotic cell death [18]. Chrysin exerts its neuroprotective effect mainly by reducing the prooxidants levels (ROS and lipid peroxidation) and augmenting the antioxidant defense factors (Figure 3) [19-23].
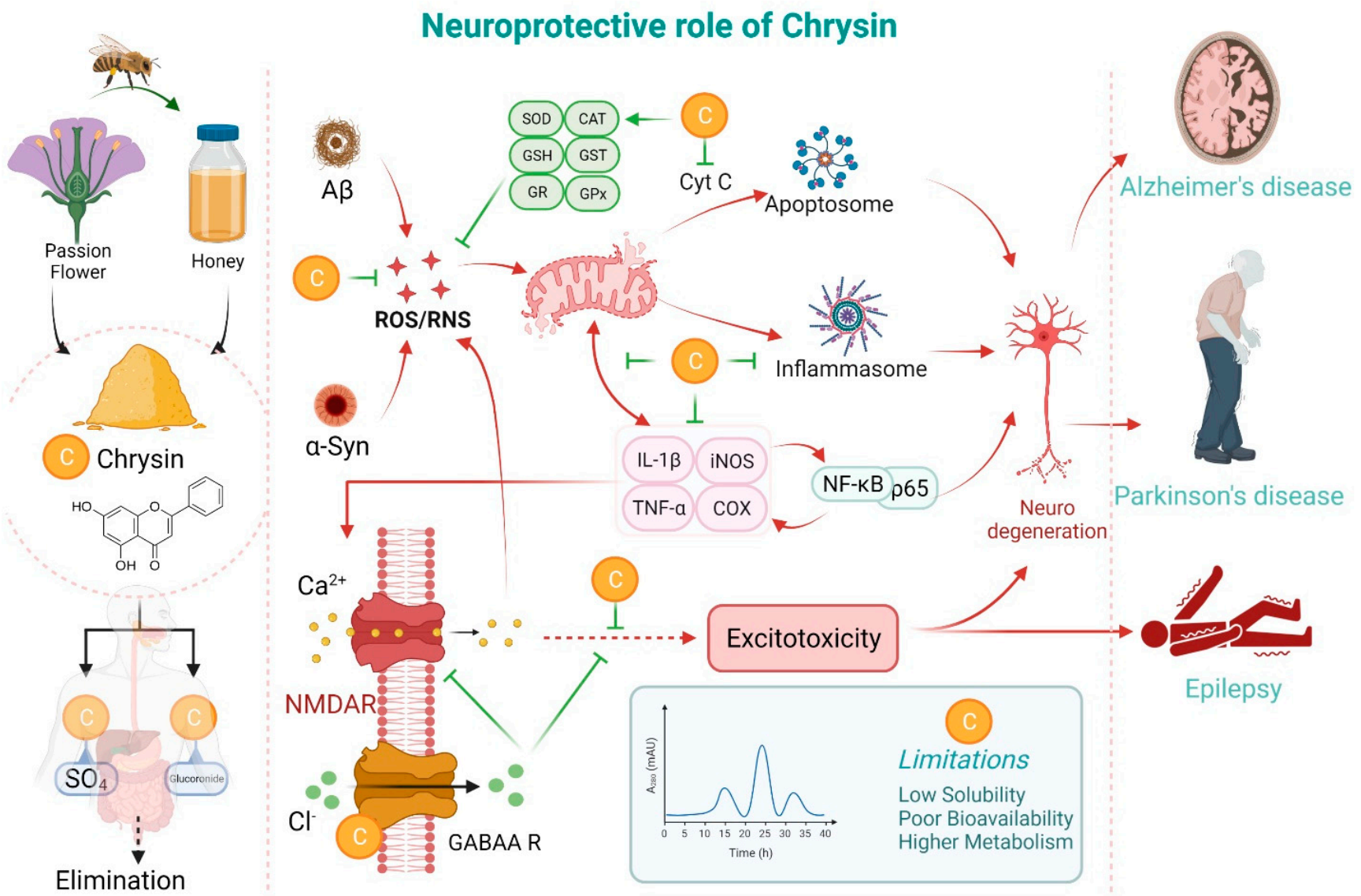

Figure 2. Effects of chrysin in the signaling networks associated with multiple neuropathological conditions.

Chrysin can also indirectly affect the oxidative stress inside the cell by inducing the expression of various key antioxidant enzymes (Figure 2), including the superoxide dismutase (SOD), catalase (CAT) and glutathione peroxidase (GPx) [22,24-26]. Amongst the three isoforms of SOD present in our body, SOD1 and SOD3 play the most important roles in the antioxidant defense mechanism $[27,28]$. SOD, CAT and GPx exhibit antioxidant functions by catalyzing the dismutation of highly reactive superoxide to the less reactive hydrogen peroxide $\left(\mathrm{H}_{2} \mathrm{O}_{2}\right)$, generating water and a dioxygen molecule from $\mathrm{H}_{2} \mathrm{O}_{2}$, while 
inhibiting lipid peroxidation [25,28-30]. Glutathione (GSH), a tripeptide abundant in the cytosol and cell organelles, is oxidized to glutathione disulfide (GSSG), and the rapid interconversion of GSH-GSSG-GSH maintains the cellular redox balance [31]. Chrysin has been shown to induce the expression of GSH, thus reducing oxidative stress [32].

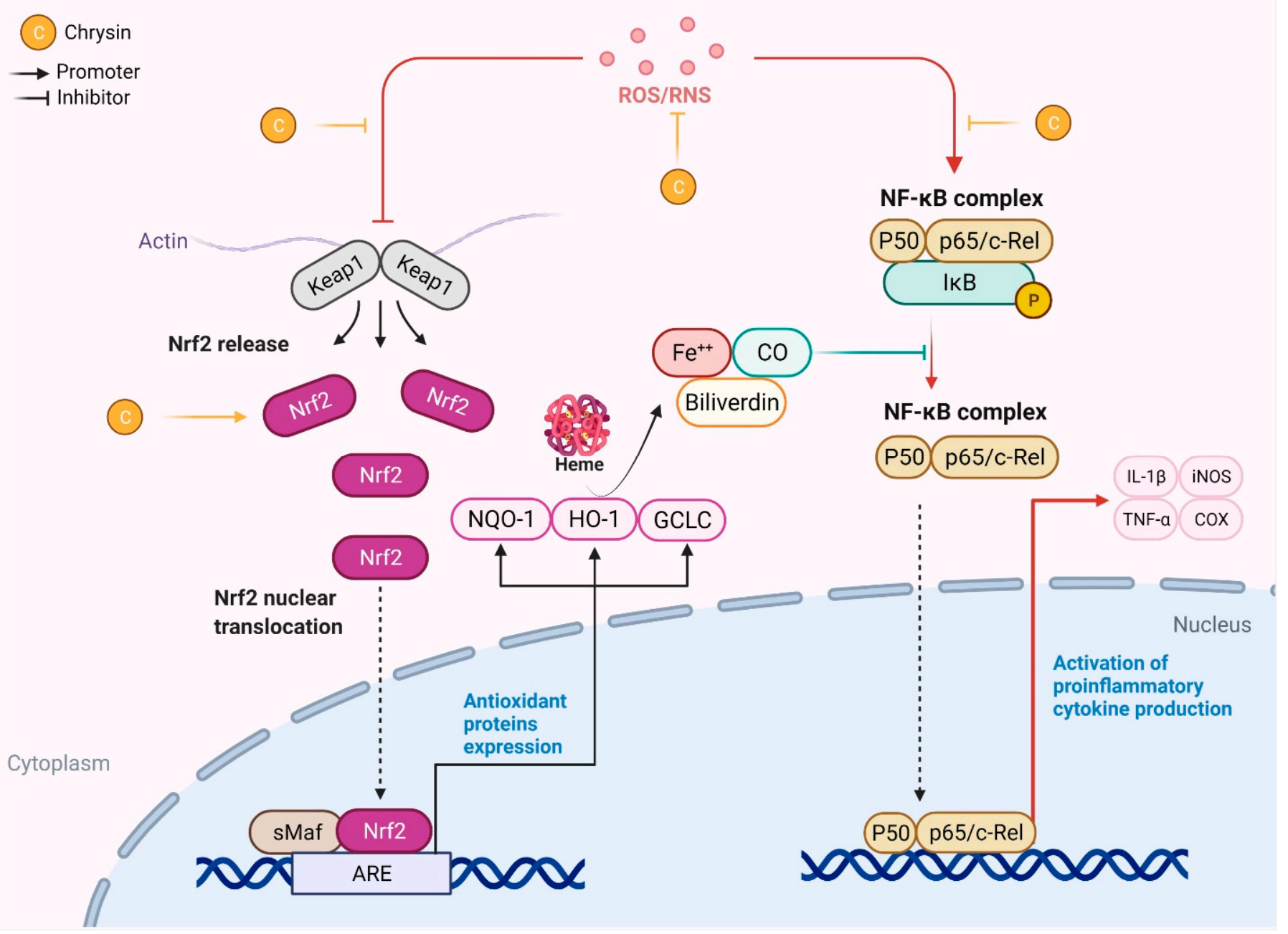

Figure 3. Modulation of the NRF-2 and NF- $\mathrm{kB}$ pathway by chrysin. ROS/RNS mediate the alteration of NRF-2 signaling and interconnect with the NF- $\mathrm{KB}$ signaling pathway. NRF-2 signaling activates the expression of antoxidant proteins viz, heme oxygenase-1 (HO-1), NAD(P)H quinone oxidoreductase 1 (NQO-1) and Glutamate-cysteine ligase catalytic subunit (GCLC, a rate-limiting enzyme for glutathione synthesis). The degradation of heme produces carbon monoxide (CO), which suppresses redox-sensitive NF- $\mathrm{kB}$ activation. The plausible sites of action of chrysin are illustrated.

\subsection{Chrysin as an Anti-Inflammatory Agent}

Inflammation is the body's natural response to injury, infection, or trauma. Upon induction, it leads to a cascade of reactions that ultimately remove invading pathogens and start the wound-healing process along with angiogenesis [33]. The first key step in neuroinflammation is the activation of microglia cells [34]. The activation of microglia occurs mainly through the activation of the c-Jun $\mathrm{N}$-terminal kinase (JNK) and the nuclear factor kappa light chain-enhancer of activated B cells (NF- $\mathrm{kB}$ ) signaling pathway [35]. NF- $\mathrm{kB}$ signaling may be aggravated by the pathogen-associated molecular pattern (PAMP)/damageassociated molecular pattern (DAMP) molecules though Toll-like receptors (TLR) and the receptor for advanced glycated end products (RAGE) [36]. The downstream effector pathways of NF- $\mathrm{kB}$ include iNOS, cyclooxygenase-2 (COX-2) and various pro-inflammatory 
cytokines $[37,38]$. The increased levels of pro-inflammatory cytokines (IL-1 $\beta$, TNF- $\alpha$, and prostaglandins) have been shown to damage the blood-brain barrier (BBB) and induce apoptosis in neuronal cells $[39,40]$. The detection of DAMPs and PAMPs by pattern recognition receptors (PRRs) such as NLRP induces the formation of protein aggregates, finally leading to the formation of inflamosomes (Figure 2) [41]. Inflamosomes further induce the secretion of pro-inflammatory cytokines, such as IL-1 $\beta$, IL-18 and pyroptosis, leading to a severe inflammatory cell response [42,43].

The anti-inflammatory activity of chrysin was shown to serve neuroprotectively after cerebral ischemia via modulation of estrogen receptors [44]. Chrysin has been also reported to modulate JNK and NF- $\mathrm{BB}$ expression and thus limit the progression of neuroinflammation [35,45-47]. It has been demonstrated to modulate the expression of NF- $\mathrm{BB}$ via the $\mathrm{PI} 3 \mathrm{~K} / \mathrm{AKT} / \mathrm{mTOR}$ and NLRP3 pathways, and to abrogate neuroinflammation [45,48-50]. Chrysin can also inhibit neuroinflammation via the mitigation of inflamosome formation by attenuating the NLRP3 signaling pathway $[48,49]$. On the other hand, chrysin directly mitigates pro-inflammatory cytokines (IL-1 $\beta$, TNF- $\alpha$, and prostaglandins) and exerts neuroprotective effects $[46,47,51]$.

\subsection{Chrysin as an Anti-Apoptotic Agent}

Apoptosis is a natural cell death process, mediated by two mechanisms, i.e., the extrinsic and intrinsic pathways (Elmore, 2007). The extrinsic pathway starts after the binding of ligands, such as Fas and TNF- $\alpha$, to the death receptors FasR and TNFR, respectively (Figure 2) [52]. This induces death signals inside the cell, leading to the activation of caspase- 8 , which further induces the activation of caspase- 3 . In turn, caspase- 3 starts an array of reactions, known as the executional pathway, which ultimately leads to apoptosis [52-55]. On the other hand, the intrinsic pathway starts with the action of various external stimuli, including radiation, toxins, hypoxia, and oxidative stress, on the cell. These increase mitochondrial membrane permeability and the release of pro-apoptotic proteins into the cytosol $[56,57]$. These events further lead to caspase- 9 activation, which ultimately activates caspase-3, thus activating the execution pathway $[52,58]$. Chrysin treatment was shown to reduce neurodegeneration by inhibiting the extrinsic, intrinsic and executionary pathways of apoptosis, and attenuating the expression of TNF- $\alpha$, caspase- $3 / 8$ and oxidative stress (Figure 2) [47,59-61].

The BCL2 protein family plays a critical role in the control and regulation of the apoptotic process. During apoptosis, the expression of anti-apoptotic proteins (Bcl-2, Bcl-x, $\mathrm{Bcl}-\mathrm{XL}, \mathrm{Bcl}-\mathrm{XS}, \mathrm{Bcl}-\mathrm{w}$ ) is decreased and the expression of pro-apoptotic proteins (Bcl-10, Bax, Bak, Bid, Bad, Bim, Bik, and Blk) is increased [52]. It had been observed that chrysin administration is accompanied by the increased expression of anti-apoptotic proteins and a decreased expression of pro-apoptotic proteins [59-63]. Chrysin acting on the dysregulated stage of apoptosis-related proteins was shown to inhibit the neuronal death of cerebellar granular neurons in mice [61].

\subsection{Chrysin as a MAO Inhibitor}

Dopamine (DA) is one of the most important neurotransmitters present in the brain. Reduced DA levels are observed in the striatum and hippocampus brain regions of PD patients. The biosynthesis of dopamine starts with tyrosine hydroxylation by tyrosine hydroxylase, and via several reactions, it gets synthesized and stored inside the synaptic vesicles of dopaminergic neurons [64]. Chrysin was shown to reduce dopamine depletion and protect against the neurodegeneration of dopaminergic neurons of the brain [61,65]. It was also observed that chrysin treatment can significantly induce dopamine level recovery in the hippocampus and the prefrontal cortex region of the brain [66].

After secretion from the neurons, dopamine gets metabolized by MAO and COMT (into DOPAC and HVA) enzymes, and to some extent by alcohol/aldehyde dehydrogenase [64]. Chrysin inhibits MAO-A and MAO-B activity [61,67] and maintains dopamine levels in the brain. Additionally, its administration protects against changes in dopamine, 
DOPAC, and HVA levels in the brain of PD-induced animals [65,68], supporting the therapeutic potential of chrysin in PD.

\subsection{Neuroprotective Role of Chrysin via a GABA Mimetic Action}

GABA is the most important inhibitory neurotransmitter in the brain. It exhibits a neuroprotective effect by inhibiting brain injury, neuronal damage, autophagy (via the upregulation of $\mathrm{Bcl}-2 / \mathrm{Bax}$ ratio and activating AKT, GSK-3 $\beta$ and ERK signaling molecules) as well as neuronal cell death [69]. In this way, GABA demonstrates a therapeutic potential in various neurological disorders $[69,70]$. Most flavonoids, including chrysin, exert a GABA mimetic effect [71]. Chrysin has been shown to modulate the GABAA receptor and thus abrogate anxiety and depression-like behavior [72-74]. Therefore, it can act neuroprotectively via the modulation of GABAergic innervation.

\section{Role of Chrysin in Different Neurological Disorders}

The neuroprotective potential of chrysin has been widely explored in several neurological disorders. A comprehensive list of studies supporting the role of chrysin in different neurological disorders is provided in Table 1.

Table 1. Experimental evidence supporting the neuroprotective role of chrysin in various neurological disorders.

\begin{tabular}{|c|c|c|c|c|c|}
\hline$\#$ & Dose and Route & $\begin{array}{l}\text { Experimental } \\
\text { Model }\end{array}$ & Animal/Cell Lines & Outcome & Ref. \\
\hline \multicolumn{6}{|c|}{ In Parkinson's Disease } \\
\hline 1. & Chrysin $(100 \mu \mathrm{M})$ & $\begin{array}{l}\text { 2,4-dinitrophenol- } \\
\text { induced } \\
\text { mitophagy }\end{array}$ & $\begin{array}{l}\text { Caenorhabditis elegans } \\
\text { (Bristol N2 wild-type, } \\
\text { ucp-4 deletion mutant, } \\
\text { pdr-1, and zdIs5) }\end{array}$ & $\begin{array}{l}\text { Chrysin served as mitochondrial } \\
\text { uncoupler and mitigated } \\
\text { neurodegeneration possibly via } \\
\text { PINK1/Parkin mitophagy }\end{array}$ & [75] \\
\hline 2. & $\begin{array}{l}\text { Chrysin }(50,100, \\
\text { and } 200 \mathrm{mg} / \mathrm{kg}, \\
\text { p.o.) for } 5 \text { days }\end{array}$ & $\begin{array}{l}\text { MPTP-induced } \\
\text { rodent model of } \\
\text { PD }\end{array}$ & $\begin{array}{l}\text { Male C57BL/6 J mice } \\
\qquad(18-22 \mathrm{~g})\end{array}$ & $\begin{array}{c}\text { Improvement in motor dysfunction and } \\
\text { dopaminergic neuroprotection in } \\
\text { nigro-striatal region, possibly by } \\
\text { mitigating oxidative stress and } \\
\text { neuroinflammation }\end{array}$ & [68] \\
\hline 3. & $\begin{array}{c}\text { Chrysin } \\
(10 \mathrm{mg} / \mathrm{kg}, \mathrm{i} . \mathrm{g} .) \text { for } \\
28 \text { days }\end{array}$ & $\begin{array}{l}\text { 6-OHDA-induced } \\
\text { rodent model of } \\
\text { PD }\end{array}$ & $\begin{array}{l}\text { Female C57B / } 6 \text { J mice } \\
\text { (30-40 g, } 20 \text { months) }\end{array}$ & $\begin{array}{l}\text { Improvement in motor and cognitive } \\
\text { functions along with reduction in } \\
\text { oxidative stress, neuroinflammation }\end{array}$ & [65] \\
\hline 4. & $\begin{array}{l}\text { Chrysin } \\
\text { (50 } \mathrm{mg} / \mathrm{Kg}, \text { i.p. }) \\
\text { for } 4 \text { weeks }\end{array}$ & $\begin{array}{l}\text { Rotenone-induced } \\
\text { rodent model of } \\
\text { PD }\end{array}$ & Sprague-Dawley rats & $\begin{array}{c}\text { Improvement in motor impairments and } \\
\text { attenuation of nigrostriatal dopaminergic } \\
\text { neurodegeneration }\end{array}$ & [76] \\
\hline 5. & $\begin{array}{l}\text { Chrysin } \\
(10 \mathrm{mg} / \mathrm{kg}, \mathrm{p} . \mathrm{o} .) \\
\text { for } 28 \text { days }\end{array}$ & $\begin{array}{l}\text { 6-OHDA-induced } \\
\text { rodent model of } \\
\text { PD }\end{array}$ & $\begin{array}{l}\text { Male C57B /6 J mice } \\
\text { (20-30g, } 90 \text { days) }\end{array}$ & $\begin{array}{l}\text { Improvement in motor functions and } \\
\text { restoration of dopaminergic neurons, } \\
\text { inflammatory cytokines, and } \\
\text { neurotrophic factors levels }\end{array}$ & [77] \\
\hline 6 & Chrysin (50 mM) & $\begin{array}{l}\mathrm{MPP}^{+} \text {-induced } \\
\text { neurotoxicity }\end{array}$ & $\begin{array}{l}\text { Primary Cerebellar } \\
\text { Granule Neuron Culture }\end{array}$ & $\begin{array}{c}\text { Neuroprotection via inhibition of } \\
\text { apoptosis by activating MEF2D via } \\
\text { AKT-GSK3 } \beta \text { signaling }\end{array}$ & [61] \\
\hline 7 & $\begin{array}{c}\text { Chrysin (10, } \\
100 \mathrm{mg} / \mathrm{kg}, \text { p.o.) }\end{array}$ & $\begin{array}{l}\text { MPTP-induced } \\
\text { rodent model of } \\
\text { PD }\end{array}$ & $\begin{array}{l}\text { Male C57BL } / 6 \text { mice } \\
(28 \pm 2 \text { g, } 8-9 \text { weeks })\end{array}$ & $\begin{array}{l}\text { Restoration of dopaminergic neuronal } \\
\text { loss via anti-apoptotic, activation of the } \\
\text { AKT-GSK3 } \beta \text { /MEF2D pathway, and } \\
\text { inhibition of MAO-B activity }\end{array}$ & [61] \\
\hline 8 & Chrysin $(25 \mu \mathrm{M})$ & $\begin{array}{l}\text { 6-OHDA-induced } \\
\text { neurotoxicity }\end{array}$ & $\begin{array}{l}\text { Rat pheochromocytoma } \\
\text { (PC12) cells }\end{array}$ & $\begin{array}{c}\text { Neuroprotection by attenuating oxidative } \\
\text { stress (NRF-2/HO-1 pathway), } \\
\text { neuroinflammation (NF-kB/iNOS } \\
\text { pathway) }\end{array}$ & [78] \\
\hline
\end{tabular}


Table 1. Cont.

\begin{tabular}{|c|c|c|c|c|c|}
\hline$\#$ & Dose and Route & $\begin{array}{l}\text { Experimental } \\
\text { Model }\end{array}$ & Animal/Cell Lines & Outcome & Ref. \\
\hline 9 & $\begin{array}{l}\text { Chrysin }(3,6 \text {, and } \\
\quad 12 \mu \mathrm{M})\end{array}$ & $\begin{array}{l}\text { 6-OHDA-induced } \\
\text { dopaminergic } \\
\text { neurotoxicity }\end{array}$ & $\begin{array}{l}\text { Zebrafish larvae } \\
\text { (AB strain) }\end{array}$ & Protection of dopaminergic neurons & [78] \\
\hline 10 & Chrysin $(40 \mu \mathrm{M})$ & $\begin{array}{l}\mathrm{MPP}^{+} \text {-induced } \\
\text { neurotoxicity }\end{array}$ & $\begin{array}{l}\text { Primary mesencephalic } \\
\text { neurons }\end{array}$ & $\begin{array}{c}\text { Neuroprotection of mesencephalic } \\
\text { dopaminergic neurons via attenuation of } \\
\text { oxidative stress and apoptosis }\end{array}$ & [79] \\
\hline \multicolumn{6}{|c|}{ In Alzheimer's Disease } \\
\hline 1 & Chrysin $(50 \mu \mathrm{M})$ & $\begin{array}{l}\text { Amylin-induced } \\
\text { amylin } \\
\text { amyloidosis }\end{array}$ & C6 Rat Glioma Cell lines & Reduction in amylin amyloidosis & [80] \\
\hline 2 & $\begin{array}{l}\text { Chrysin } \\
(10 \mathrm{mg} / \mathrm{kg} \text {, p.o. }) \\
\text { for } 3 \text { months }\end{array}$ & $\begin{array}{l}\text { Zinc-induced } \\
\text { cognitive } \\
\text { impairment and } \\
\text { amyloidosis }\end{array}$ & Male Swiss mice & $\begin{array}{l}\text { Improvement in cognitive functions and } \\
\text { neuroprotection of hippocampal neurons }\end{array}$ & [81] \\
\hline 3 & $\begin{array}{l}\text { Chrysin loaded } \\
\text { lipid-core nano } \\
\text { capsules ( } 1 \text { and } \\
5 \text { mg/kg., p.o.) for } \\
14 \text { days }\end{array}$ & $\begin{array}{l}\mathrm{A} \beta_{1-42} \text {-induced } \\
\text { animal model of } \\
\text { AD }\end{array}$ & $\begin{array}{c}\text { Female Swiss mice } \\
\text { (30-35 g, 18-22 months) }\end{array}$ & $\begin{array}{l}\text { Improvement in learning and memory } \\
\text { impairment via attenuation of oxidative } \\
\text { stress and neuroinflammation }\end{array}$ & [82] \\
\hline 4 & $\begin{array}{l}\text { Chrysin loaded } \\
\text { magnetic } \\
\text { PEGylated silica } \\
\text { nanospheres } \\
(30 \mu \mathrm{M})\end{array}$ & $\begin{array}{l}\text { A } \beta \text {-induced } \\
\text { amyloidosis in } \\
\text { hippocampal } \\
\text { culture model }\end{array}$ & $\begin{array}{l}\text { Sprague-Dawley rat } \\
\text { (neonates) }\end{array}$ & $\begin{array}{c}\text { Improved antioxidant profile and } \\
\text { protection against } A \beta \text {-induced oxidative } \\
\text { stress }\end{array}$ & [83] \\
\hline 5 & $\begin{array}{l}\text { Chrysin } \\
(50 \mathrm{mg} / \mathrm{kg}) \text { for } \\
21 \text { days }\end{array}$ & $\begin{array}{l}\text { Acrylamide or } \\
\text { gamma-irradiation } \\
\text { induced } \\
\text { neurotoxicity }\end{array}$ & $\begin{array}{l}\text { Male Wister rats } \\
\quad(120-150 \mathrm{~g})\end{array}$ & $\begin{array}{l}\text { Neuroprotective effect via attenuation of } \\
\text { oxidative stress, amyloidosis, and } \\
\text { apoptosis }\end{array}$ & [84] \\
\hline 6 & $\begin{array}{l}\text { Chrysin loaded } \\
\text { sloid lipid } \\
\text { nanoparticles }(5 \\
\text { 10, 50, } 100 \mathrm{mg} / \mathrm{kg} \\
\text { p.o.) for } 21 \text { days }\end{array}$ & $\begin{array}{l}\mathrm{A} \beta_{25-35} \text {-induced } \\
\text { rodent model of } \\
\mathrm{AD}\end{array}$ & $\begin{array}{l}\text { Male Sprague-Dawley } \\
\quad \text { rats }(250-300 \mathrm{~g})\end{array}$ & $\begin{array}{c}\text { Improved memory impairment and } \\
\text { amelioration of hippocampal neuronal } \\
\text { loss possibly via mitigation of } \\
\text { neuronal loss }\end{array}$ & [22] \\
\hline \multicolumn{6}{|c|}{ In Epilepsy } \\
\hline 1 & $\begin{array}{l}\text { Chrysin-loaded } \\
\text { PLGA nanoparticle } \\
(5 \text { and } 10 \mu \mathrm{g} / \mathrm{mL})\end{array}$ & $\begin{array}{l}\text { PTZ-induced } \\
\text { kindling }\end{array}$ & Wistar rats & $\begin{array}{l}\text { Anticonvulsant effect through mitigating } \\
\text { oxidative stress via the NRF2/HO-1 } \\
\text { pathway }\end{array}$ & [15] \\
\hline 2 & $\begin{array}{l}\text { Chrysin }(2.5,5 \text {, and } \\
10 \mathrm{mg} / \mathrm{kg} ; \text { p.o. })\end{array}$ & $\begin{array}{l}\text { PTZ-induced } \\
\text { convulsions }\end{array}$ & $\begin{array}{l}\text { Male Charles Foster rats } \\
\qquad(180-220 \mathrm{~g})\end{array}$ & $\begin{array}{l}\text { Anticonvulsant effect possibly via } \\
\text { alleviation of oxidative stress in } \\
\text { hippocampus and cortex }\end{array}$ & [85] \\
\hline 3 & $\begin{array}{l}\text { Hydroethanolic } \\
\text { extract of Passiflora } \\
\text { incarnata }(150,300 \text {, } \\
\text { and } 600 \mathrm{mg} / \mathrm{kg} \text {, } \\
\text { i.p.) for } 11 \text { days }\end{array}$ & $\begin{array}{l}\text { PTZ-induced } \\
\text { kindling }\end{array}$ & $\begin{array}{l}\text { Male Swiss mice } \\
\qquad(20-30 \mathrm{~g})\end{array}$ & $\begin{array}{l}\text { Presence of chrysin in the extract and } \\
\text { anticonvulsant, antidepressant effects }\end{array}$ & [86] \\
\hline 4 & $\begin{array}{l}\text { Extract of Passiflora } \\
\text { incarnata }(150,300 \\
\text { and } 600 \mathrm{mg} / \mathrm{kg} ; \\
\text { i.p.) }\end{array}$ & $\begin{array}{l}\text { PTZ-induced } \\
\text { convulsions }\end{array}$ & $\begin{array}{l}\text { Male Swiss mice } \\
\qquad(25-35 \mathrm{~g})\end{array}$ & $\begin{array}{l}\text { Presence of chrysin in the extracts and } \\
\text { anticonvulsant effect of the extracts }\end{array}$ & [87] \\
\hline
\end{tabular}


Table 1. Cont.

\begin{tabular}{|c|c|c|c|c|c|}
\hline$\#$ & Dose and Route & $\begin{array}{c}\text { Experimental } \\
\text { Model }\end{array}$ & Animal/Cell Lines & Outcome & Ref. \\
\hline 5 & $\begin{array}{l}\text { Chrysin }(40 \mu g, \\
\text { i.c.v. })\end{array}$ & $\begin{array}{l}\text { PTZ-induced } \\
\text { convulsions }\end{array}$ & Swiss mice (22-28 g) & $\begin{array}{l}\text { Anticonvulsant effect via activation of } \\
\text { benzodiazepine receptors }\end{array}$ & [88] \\
\hline \multicolumn{6}{|c|}{ Chrysin in Traumatic Brain Injury } \\
\hline 1 & $\begin{array}{l}\text { Chrysin }(25,50 \text { and } \\
100 \mathrm{mg} / \mathrm{kg} \text {, p.o. })\end{array}$ & $\begin{array}{l}\text { Closed head } \\
\text { weight-drop- } \\
\text { induced rodent } \\
\text { model TBI }\end{array}$ & $\begin{array}{l}\text { Male Wistar rats } \\
\quad(250-300 \mathrm{~g})\end{array}$ & $\begin{array}{l}\text { Improved neurobehavioral impairments } \\
\text { possibly via modulation of inflammation } \\
\text { and apoptosis }\end{array}$ & [89] \\
\hline 2 & $\begin{array}{l}\text { Chrysin }(25,50 \text { and } \\
100 \mathrm{mg} / \mathrm{kg} \text {, p.o. })\end{array}$ & $\begin{array}{l}\text { Closed head } \\
\text { weight-drop- } \\
\text { induced rodent } \\
\text { model TBI }\end{array}$ & $\begin{array}{l}\text { Adult male Wistar rats } \\
\qquad(250-300 \mathrm{~g})\end{array}$ & $\begin{array}{l}\text { Improved motor coordination and } \\
\text { memory impairment possibly via } \\
\text { anti-oxidant and anti-apoptotic effects }\end{array}$ & [62] \\
\hline \multicolumn{6}{|c|}{ Chrysin in Ischemic Brain Injury } \\
\hline 1 & $\begin{array}{l}\text { Chrysin }(10, \text { and } \\
20 \mathrm{mg} / \mathrm{kg}) \text { for } \\
7 \text { days }\end{array}$ & $\begin{array}{l}\text { Middle cerebral } \\
\text { artery } \\
\text { occlusion-induced } \\
\text { cerebral is- } \\
\text { chemia/reperfusion } \\
\text { injury model }\end{array}$ & $\begin{array}{l}\text { Male Sprague-Dawley } \\
\quad \text { rats }(250-280 \mathrm{~g})\end{array}$ & $\begin{array}{l}\text { Reduction in ischemia/reperfusion injury } \\
\text { in brain, possibly via alleviation of } \\
\text { proinflammatory cytokine release and } \\
\text { improvement of antioxidant defense by } \\
\text { activating the PI3K/Akt/mTOR pathway }\end{array}$ & [45] \\
\hline 2 & $\begin{array}{l}\text { Chrysin }(10,30, \\
\text { and } 100 \mathrm{mg} / \mathrm{kg} \text {, } \\
\text { p.o.) for } 21 \text { days }\end{array}$ & $\begin{array}{l}\text { Bilateral common } \\
\text { carotid arteries } \\
\text { occlusion model of } \\
\text { cerebral ischemia } \\
\text { reperfusion injury }\end{array}$ & $\begin{array}{l}\text { Male Wistar rats } \\
(250-300 \mathrm{~g}, 6 \text { month })\end{array}$ & $\begin{array}{l}\text { Improvement in cognitive impairment } \\
\text { and restoration of hippocampal neurons } \\
\text { possibly by reducing oxidative stress and } \\
\text { PGE2 levels }\end{array}$ & [90] \\
\hline 3 & $\begin{array}{l}\text { Chrysin }(10,30, \\
\text { and } 100 \mathrm{mg} / \mathrm{kg}, \\
\text { p.o.) for } 21 \text { days }\end{array}$ & $\begin{array}{l}\text { Bilateral common } \\
\text { carotid arteries } \\
\text { occlusion model of } \\
\text { cerebral ischemia } \\
\text { reperfusion injury }\end{array}$ & $\begin{array}{l}\text { Male Wistar rats } \\
\quad(250-300 \mathrm{~g})\end{array}$ & $\begin{array}{c}\text { Improvement in cognitive impairment } \\
\text { possibly by alleviating } \\
\text { neuroinflammation }\end{array}$ & [91] \\
\hline 4 & $\begin{array}{l}\text { Chrysin } \\
\text { (30 } \mathrm{mg} / \mathrm{kg} \text {, p.o.) } \\
\text { for } 14 \text { days }\end{array}$ & $\begin{array}{l}\text { Bilateral common } \\
\text { carotid arteries } \\
\text { occlusion model of } \\
\text { cerebral ischemia } \\
\text { reperfusion injury }\end{array}$ & $\begin{array}{l}\text { Male Wistar rats } \\
\quad(200-250 \mathrm{~g})\end{array}$ & $\begin{array}{l}\text { Neuroprotection against ischemia } \\
\text { reperfusion injury possibly by } \\
\text { attenuating oxidative stress }\end{array}$ & [92] \\
\hline 5 & $\begin{array}{l}\text { Chrysin } \\
(50 \mathrm{mg} / \mathrm{kg}, \mathrm{p} . \mathrm{o} .) \\
\text { for } 10 \text { days }\end{array}$ & $\begin{array}{l}\text { Bilateral common } \\
\text { carotid arteries } \\
\text { occlusion model of } \\
\text { cerebral ischemia } \\
\text { reperfusion injury }\end{array}$ & $\begin{array}{l}\text { Male C57BL / } 6 \text { J mice } \\
\quad(18-22 \mathrm{~g})\end{array}$ & $\begin{array}{c}\text { Reduction in degenerative changes in } \\
\text { neurons possibly by mitigating oxidative } \\
\text { stress }\end{array}$ & [93] \\
\hline 6 & $\begin{array}{l}\text { Chrysin } \\
(75 \mathrm{mg} / \mathrm{kg}, \mathrm{p} . \mathrm{o} .) \\
\text { for } 7 \text { days }\end{array}$ & $\begin{array}{l}\text { Middle cerebral } \\
\text { artery } \\
\text { occlusion-induced } \\
\text { ischemia } \\
\text { reperfusion injury }\end{array}$ & $\begin{array}{l}\text { Male C57 / BL6 mice } \\
\quad(10-12 \text { weeks })\end{array}$ & $\begin{array}{l}\text { Reduction in neurological deficit scores } \\
\text { and infarct volumes, possibly via } \\
\text { inhibition of neuroinflammation (by } \\
\text { suppression of NF- } \mathrm{B}, \mathrm{COX}-2 \text {, and iNOS } \\
\text { expression) }\end{array}$ & [94] \\
\hline 7 & $\begin{array}{l}\text { Chrysin ( } 30, \text { and } \\
100 \mathrm{mg} / \mathrm{kg} ; \mathrm{i} . \mathrm{g} .) \\
\quad \text { for } 26 \text { days }\end{array}$ & $\begin{array}{l}\text { Bilateral common } \\
\text { carotid arteries } \\
\text { occlusion model of } \\
\text { cerebral ischemia } \\
\text { reperfusion injury }\end{array}$ & $\begin{array}{l}\text { Male Wistar rats, } \\
\quad(330-350 \mathrm{~g})\end{array}$ & $\begin{array}{c}\text { Improvement in dementia and } \\
\text { neurodegeneration possibly via } \\
\text { attenuation of oxidative stress and } \\
\text { neuroinflammation }\end{array}$ & [95] \\
\hline
\end{tabular}


Table 1. Cont.

\begin{tabular}{|c|c|c|c|c|c|}
\hline$\#$ & Dose and Route & $\begin{array}{l}\text { Experimental } \\
\text { Model }\end{array}$ & Animal/Cell Lines & Outcome & Ref. \\
\hline \multicolumn{6}{|c|}{ Chrysin in gliomas } \\
\hline 1 & $\begin{array}{l}\text { Chrysin }(5,30,60 \\
120, \text { and } 240 \mu \mathrm{M})\end{array}$ & $\begin{array}{l}\text { Human } \\
\text { glioblastoma cell } \\
\text { lines }\end{array}$ & T98, U251, U87 cells & $\begin{array}{c}\text { Anticancer activity in glioblastoma cell } \\
\text { lines possibly via the ERK/Nrf2 } \\
\text { signaling pathway }\end{array}$ & [96] \\
\hline 2 & $\begin{array}{c}\text { Chrysin }(10,20,40, \\
80 \text { and } 120 \mu \mathrm{M}) \\
\text { and Cisplatin }(0.5, \\
1.0 \text { and } 2.0 \mu \mathrm{M}) \\
\text { combination }\end{array}$ & $\begin{array}{l}\text { Human glioma cell } \\
\text { lines }\end{array}$ & U87 cells & $\begin{array}{l}\text { Potentiation of antiproliferative effect of } \\
\text { Cisplatin }\end{array}$ & [97] \\
\hline 3 & Chrysin $(50 \mu \mathrm{M})$ & $\begin{array}{l}\text { Human } \\
\text { glioblastoma cell } \\
\text { lines }\end{array}$ & GL-15 and U251 cells & $\begin{array}{l}\text { Damaged mitochondria, and rough } \\
\text { endoplasmic reticulum, apoptosis, and } \\
\text { reduction in MMP-2 expression }\end{array}$ & [98] \\
\hline 4 & Chrysin $(100 \mu \mathrm{M})$ & $\begin{array}{l}\text { Human } \\
\text { glioblastoma cell } \\
\text { lines }\end{array}$ & $\begin{array}{l}\text { GBM8901 glioblastoma } \\
\text { cells }\end{array}$ & $\begin{array}{l}\text { Induction of apoptosis and suppression } \\
\text { of migration and invasion. Inhibition of } \\
\text { temozolomide-induced autophagy and } \\
\text { O6-methylguanine-DNA }\end{array}$ & [99] \\
\hline 5 & $\begin{array}{l}\text { Chrysin }(10,30, \\
\text { and } 50 \mu \mathrm{M})\end{array}$ & Rat glioma cell line & C6 glioma cells & $\begin{array}{l}\text { Induction of G1 phase cell cycle arrest } \\
\text { through induction of p } 21^{\text {Waf1/Cip } 1} \text { and } \\
\text { inhibition of proteasome activity }\end{array}$ & [100] \\
\hline \multicolumn{6}{|c|}{ Chrysin in MS } \\
\hline 1 & $\begin{array}{c}\text { Chrysin } \\
(20 \mathrm{mg} / \mathrm{kg}, \mathrm{i} . \mathrm{g} .) \text { for } \\
25 \text { days }\end{array}$ & $\begin{array}{l}\text { Myelin } \\
\text { oligodendrocyte } \\
\text { glycoprotein- } \\
\text { induced } \\
\text { EAE }\end{array}$ & $\begin{array}{l}\text { Male C57BL / } 6 \text { mice } \\
\quad(20-25 \mathrm{~g})\end{array}$ & $\begin{array}{l}\text { Reduction in HDAC activity, GSK-3 } \beta \text { and } \\
\text { proinflammatory cytokine release }\end{array}$ & [101] \\
\hline 2 & $\begin{array}{l}\text { Chrysin } \\
\text { (100 } \mathrm{mg} / \mathrm{kg}, \mathrm{i.g} .) \\
\text { for } 3 \text { days }\end{array}$ & $\begin{array}{l}\text { Myelin } \\
\text { oligodendrocyte } \\
\text { glycoprotein- } \\
\text { induced } \\
\text { EAE }\end{array}$ & $\begin{array}{l}\text { Female C57BL } / 6 \text { mice } \\
\qquad(6-8 \text { weeks })\end{array}$ & $\begin{array}{l}\text { Amelioration of EAE and } \\
\text { anti-inflammatory and immune } \\
\text { suppressive effects via suppression of } \\
\text { dendritic cells and Th1 cells }\end{array}$ & [102] \\
\hline \multicolumn{6}{|c|}{ Miscellaneous } \\
\hline 1 & Chrysin $(0.05 \mathrm{mM})$ & $\begin{array}{l}\text { Diclofenac- } \\
\text { induced } \\
\text { neurotoxicity }\end{array}$ & SH-SY5Y cells & $\begin{array}{l}\text { Neuroprotective effect of mitigating } \\
\text { oxidative stress and apoptosis }\end{array}$ & {$[60]$} \\
\hline 2 & Chrysin $(400 \mu \mathrm{M})$ & $\begin{array}{l}\text { Cyclophosphamide- } \\
\text { induced } \\
\text { neurotoxicity }\end{array}$ & SH-SY5Y cells & $\begin{array}{l}\text { Neuroprotection via suppression of } \\
\text { oxidative stress and apoptotic cell death }\end{array}$ & [59] \\
\hline 2 & $\begin{array}{l}\text { Chrysin }(5, \text { and } \\
10 \mu \mathrm{M})\end{array}$ & $\begin{array}{l}\text { LPS-induced } \\
\text { inflammation }\end{array}$ & $\begin{array}{l}\text { BV2 microglia cells and } \\
\text { Primary mouse } \\
\text { microglia cells }\end{array}$ & \multirow{2}{*}{$\begin{array}{l}\text { Suppression of neuroinflammation by } \\
\text { downregulating NF- } \mathrm{kB} \text { /TRAF6 pathway } \\
\text { and upregulating zinc figure protein } \mathrm{A} 20\end{array}$} & \multirow{2}{*}{103} \\
\hline 3 & $\begin{array}{c}\text { Chrysin (25 and } \\
50 \mathrm{mg} / \mathrm{kg} \text {, p.o. }) \text { for } \\
4 \text { days }\end{array}$ & $\begin{array}{l}\text { LPS-induced neu- } \\
\text { roinflammation }\end{array}$ & $\begin{array}{l}\text { Male Balb/c mice } \\
(20 \pm 2 \mathrm{~g}, 8 \text { weeks })\end{array}$ & & \\
\hline 4 & $\begin{array}{c}\text { Chrysin } \\
(20 \mathrm{mg} / \mathrm{kg}, \mathrm{i} . \mathrm{g} .) \text { for } \\
28 \text { days }\end{array}$ & $\begin{array}{l}\text { Methimazole } \\
\text { induced } \\
\text { hypothyroidism } \\
\text { and associated } \\
\text { neurobehavioral } \\
\text { impairments }\end{array}$ & $\begin{array}{l}\text { Female C57BL } / 6 \text { mice } \\
\qquad(3-4 \text { months })\end{array}$ & $\begin{array}{l}\text { Improvement in depression-like behavior } \\
\text { via improvement of cortical and } \\
\text { hippocampal serotonin levels and } \\
\text { hippocampal dopamine levels }\end{array}$ & [66] \\
\hline
\end{tabular}


Table 1. Cont.

\begin{tabular}{|c|c|c|c|c|c|}
\hline$\#$ & Dose and Route & $\begin{array}{l}\text { Experimental } \\
\text { Model }\end{array}$ & Animal/Cell Lines & Outcome & Ref. \\
\hline 5 & $\begin{array}{c}\text { Chrysin }(60,80, \\
100,150 \text { and } \\
200 \mu \mathrm{g} / \mathrm{mL})\end{array}$ & $\begin{array}{l}\text { LPS-induced neu- } \\
\text { roinflammation }\end{array}$ & $\begin{array}{c}\text { RAW264.7 } \\
\text { macrophage cells }\end{array}$ & $\begin{array}{l}\text { Reduction in inflammatory response by } \\
\text { blocking the JAK-STAT pathway } \\
\text { mediated by ROS }\end{array}$ & [51] \\
\hline 6 & $\begin{array}{c}\text { Chrysin } \\
(10-100 \mu \mathrm{M})\end{array}$ & $\begin{array}{l}\text { LPS-induced } \\
\text { in vitro study }\end{array}$ & $\begin{array}{c}\text { Mouse cerebral vascular } \\
\text { endothelial } \\
\text { (bEnd.3) cells }\end{array}$ & $\begin{array}{l}\text { Reduction in VCAM-1 expression by } \\
\text { inhibition of NF- } \mathrm{B} \text { /MAPK pathway } \\
\text { resulted in anti-inflammatory effect }\end{array}$ & [46] \\
\hline 7 & $\begin{array}{c}\text { Chrysin } \\
(100 \mathrm{mg} / \mathrm{kg}, \text { p.o. })\end{array}$ & $\begin{array}{l}\text { Ammonium } \\
\text { chloride-induced } \\
\text { neuroinflamma- } \\
\text { tion }\end{array}$ & Male Wistar rats & $\begin{array}{l}\text { Attenuation of neuroinflammation by } \\
\text { reducing expression of pro-inflammatory } \\
\text { markers (TNF- } \alpha \text {, IL-1 } \beta \text {, IL-6, NF- } k B \text {, } \\
\text { iNOS and COX-2) in the brain }\end{array}$ & [104] \\
\hline 8 & $\begin{array}{l}\text { Chrysin } \\
(50 \mathrm{mg} / \mathrm{kg}, \mathrm{p} . \mathrm{o} .) \\
\text { for } 14 \text { days }\end{array}$ & $\begin{array}{l}\text { 3-nitro propionic } \\
\text { acid-induced } \\
\text { neurotoxicity }\end{array}$ & $\begin{array}{l}\text { Male Wistar rats } \\
\quad(250-300 \mathrm{~g})\end{array}$ & $\begin{array}{l}\text { Improvement in neurobehavioral } \\
\text { impairments, mitochondrial dysfunction, } \\
\text { oxidative stress, and apoptosis }\end{array}$ & [63] \\
\hline 9 & $\begin{array}{l}\text { Chrysin } \\
(10 \mathrm{mg} / \mathrm{kg} \text {, p.o.) } \\
\text { for } 60 \text { days }\end{array}$ & $\begin{array}{l}\text { Age-related } \\
\text { memory decline in } \\
\text { mice }\end{array}$ & $\begin{array}{l}\text { Male Swiss Mice ( } 3 \text { and } \\
20 \text { months) }\end{array}$ & $\begin{array}{l}\text { Improvement in age-related memory } \\
\text { decline by attenuating oxidative stress } \\
\text { and } \mathrm{Na}^{+} / \mathrm{K}^{+} \text {ATPase activity in } \\
\text { prefrontal cortex and hippocampus }\end{array}$ & [26] \\
\hline 10 & $\begin{array}{l}\text { Chrysin }(5 \text { and } \\
20 \mathrm{mg} / \mathrm{kg} \text {, p.o. }) \text { for } \\
28 \text { days }\end{array}$ & $\begin{array}{l}\text { Chronic } \\
\text { unpredictable mild } \\
\text { stress }\end{array}$ & $\begin{array}{l}\text { Female C57B / } 6 \text { J mice } \\
\text { (20-25 g, } 90 \text { days })\end{array}$ & $\begin{array}{l}\text { Alleviation of depression-like symptoms } \\
\text { possibly by upregulation of BDNF, NGF } \\
\text { levels, antioxidant defense factor (GPx, } \\
\text { GR, Catalase) and reduction in ROS level } \\
\text { and } \mathrm{Na}^{+} / \mathrm{K}^{+} \text {ATPase activity }\end{array}$ & [105] \\
\hline 11 & $\begin{array}{l}\text { Chrysin ( } 30 \text { and } \\
100 \mathrm{mg} / \mathrm{kg} \text {, i.g.) } \\
\quad \text { for } 26 \text { days }\end{array}$ & $\begin{array}{l}\text { Weight-drop } \\
\text { method-induced } \\
\text { spinal cord injury } \\
\text { model }\end{array}$ & Wistar rats (230-250 g) & $\begin{array}{l}\text { Augmentation in neuronal recovery and } \\
\text { reduction in pro-inflammatory markers } \\
\text { and iNOS expression }\end{array}$ & [106] \\
\hline 12 & $\begin{array}{l}\text { Chrysin in vitro } \\
(0.5-5 \mu \mathrm{M}) \text { for } \\
12 \text { and } 24 \mathrm{~h}\end{array}$ & $\begin{array}{l}\text { Acrylamide- } \\
\text { induced } \\
\text { neurotoxicity }\end{array}$ & PC12 cells & Neuroprotection & [107] \\
\hline 13 & $\begin{array}{l}\text { Chrysin in vivo } \\
(12.5,25, \text { and } \\
50 \mathrm{mg} / \mathrm{kg})\end{array}$ & $\begin{array}{l}\text { Acrylamide- } \\
\text { induced toxicity } \\
\text { in vivo }\end{array}$ & $\begin{array}{l}\text { Male Wistar rats } \\
\quad(230-250 \mathrm{~g})\end{array}$ & Reduction in gait abnormality & [107] \\
\hline
\end{tabular}

\subsection{Chrysin in $A D$}

$\mathrm{AD}$ is one of the most common progressive neurodegenerative disorders, characterized by dementia, while the oligomerization of amyloid-beta $(\mathrm{A} \beta)$ and the hyperphosphorylation of tau protein are considered as important pathological hallmarks. These abnormal protein aggregates initiate a variety of cellular responses (neuroinflammation, mitochondrial dysfunction, epigenetic alterations, and BBB changes), and eventually lead to neuronal death [108].

Studies have shown that chrysin may exert beneficial effects in AD disease models. The treatment of animals with chrysin-loaded magnetic PEGylated silica nanospheres has attenuated $\mathrm{A} \beta$-induced memory impairment, possibly through the reduction of hippocampal lipid peroxidation levels and the elevation of antioxidant molecules (GSH, GPX, catalase, SOD, GSH), enabling neuroprotection $[22,83,109]$. In another study, free chrysin as well as CN-SLN were demonstrated to reverse learning impairment, along with a reduction in the neuroinflammation induced by $\mathrm{A} \beta$, by lowering the expressions of IL- $1 \beta$, IL-10 and TNF- $\alpha$ in the brain [82]. In MTZ-induced hypothyroid and associated dementia, chrysin treatment was demonstrated to reverse memory loss by overturning the decreased glutamate level and $\mathrm{Na}^{+} / \mathrm{K}^{+}$-ATPase activity [110]. 


\subsection{Chrysin in $P D$}

$\mathrm{PD}$ is the second most common neurodegenerative disorder, characterized by motor (bradykinesia, rigidity, tremors) and non-motor manifestations (pain, bladder and bowel disorders, depression). The chronic condition often disables the patient with shuffling gait, improper balance, and cognitive impairment [5,81].

Chrysin was shown to exhibit beneficial effects in various experimental models of PD. In the 1-methyl-4-phenyl-1, 2, 3, 6-tetrahydropyridine (MPTP)-induced experimental model of PD, chrysin treatment reduced the loss of dopaminergic neurons, possibly by mitigating apoptosis via the modulation of the AKT/GSK3 $\beta$ pathway and by restoring the imbalance in BCL2 family proteins [61]. Chrysin treatment has also caused a reduction in 6-hydroxydopamine (6-OHDA)-induced dopaminergic neuronal loss in substantia nigra pars compacta dopaminergic neurons, by mitigating oxidative stress through the activation of the NRF2/HO-1 pathway and neuroinflammation [65,78]. Chrysin restored striatal dopaminergic neuronal loss and improved the dopamine turnover in the striatum [77], supporting the protective effect of chrysin on motor functions [76].

\subsection{Chrysin in Epilepsy}

Epilepsy is a devastating neurological disorder characterized by unprovoked recurrent seizures, which might be attributed to aberrant neuronal activity. The pathomechanism of epilepsy is not yet fully understood. However, the imbalance in excitatory and inhibitory neurotransmission in the brain possibly contributes to the generation and propagation of seizures. In addition, alterations in the ion channels' expression in the brain are considered as a plausible underlying cause [111-113].

The hydroethanolic extract of Passiflora incarnata L., its aqueous form (PIAE), as well as the hydroethanolic (PIHE) extract of Passiflora incarnata contain chrysin as an active ingredient. Their administration was shown to reduce pentylenetetrazol (PTZ)induced seizure onset time, along with the severity and immobility period [86,87]. The administration of the ethanolic extract of Pyrus pashia fruits (containing chrysin as an active ingredient) exhibited anticonvulsant effects in PTZ-induced convulsions, along with antioxidant effects [85].

\subsection{Chrysin in MS}

MS is a relatively common disease of the central nervous system, characterized by inflammatory demyelination. The myelin sheath is essential for the protection of neuronal axons in the brain and the spinal cord, and MS is considered as an autoimmune disease. The animal model used for mimicking MS pathogenesis and the study of therapeutic interventions is the experimental autoimmune encephalomyelitis (EAE) model. The administration of chrysin in MS animal disease models was shown to improve clinical scores. Moreover, histone deacetylase inhibitors (HDACi) have been proposed as potential effective agents in neuroinflammatory diseases, including MS, due to their neuroprotective and immunosuppressive effects. Chrysin can block HDAC expression and reduce neuroinflammation in an EAE model [114]. It also causes weight loss, lowering cytotoxicity in animals, suggesting that HDAC inhibition by chrysin may be beneficial in the rodent EAE model [93].

Chrysin may also have significant effects on human DCs (dendritic cells). It can further eliminate the monocytes in peripheral blood mononuclear cells (PBMCs) in vitro and inhibit inflammatory cytokine production, along with the metabolic activity of PBMCs stimulated by lipopolysaccharide (LPS). Chrysin was further shown to induce phenotypic and functional changes in DCs [94]. Collectively, these findings suggest that chrysintreated m-DCs may have the potential to reduce HLA-DR costimulatory molecules and induce $\mathrm{T}$ cell proliferation. Therefore, it has been proposed that the inhibitory effects of chrysin on antigen presentation may play a vital role in the pathogenesis of EAE and MS [109]. Additionally, chrysin has been reported to inhibit vascular cell adhesion molecule1 expression via the inhibition of NF-KB/MAPK signaling, which is also significantly implicated in MS pathogenesis [46]. 


\subsection{Chrysin in Traumatic and Ischemic Brain Injury}

TBI is considered one of the common etiologies of neurological disorders. There are various clinical features of TBI, including reduced alertness, attention, memory loss, vison impairment, muscle weakness, etc. Treatment with chrysin was shown to reduce TBI-induced oculomotor dysfunction and memory impairment by inhibiting neuroinflammation and apoptosis via the upregulation of the Bcl-2 family and the downregulation of the Bax protein [62,89]. In another study, chrysin supported the alleviation of TBIrelated anxiety and depression-like behavior. Furthermore, treatment with chrysin (10 and $20 \mathrm{mg} / \mathrm{kg}$ ) was demonstrated to reduce brain edema after ischemic stroke [89]. Chrysin further reduced post-ischemic injury by alleviating the expression of pro-inflammatory cytokines (TNF- $\alpha$ and IL-10), as well as reducing pro-apoptotic (Bax) and augmenting anti-apoptotic (Bcl2) protein expression, thus exerting neuroprotective effects $[45,89]$.

\subsection{Chrysin in Gliomas}

Gliomas are the most common brain tumors caused by the aberrant proliferation of glial cells, occurring both in the brain and the spinal cord. Glial cells, including astrocytes, oligodendrocytes, and microglia, support neuronal function. It has been shown that compounds found in propolis, such as CAPE, and chrysin may inhibit the NF- $\mathrm{kB}$ signaling pathway, a key signaling axis in glioma development and progression [115]. Moreover, it has been observed that the ethanolic extract of propolis interacts with the TMZ complex and may inhibit glioblastoma progression [115].

Chrysin treatment arrests the glioma cell cycle in G1 phase by increasing P21 (waf1/cip1) protein and activating P38-MAPK [100]. Chrysin combined with pine-needle extracts may regulate O-6-Methylguanine-DNA Methyltransferase (MGMT) suppression and AKT signaling, which play key roles in gliomagenesis [99]. Chrysin exhibited greater antiglioblastoma activity compared to other compounds (PWE, pinocembrin, tiliroside) in GBM8901 cells. It was associated with reduced growth in the range of 25 to $100 \mu \mathrm{M}$ in a time-dependent manner in GBM8901 cells [99]. However, in contrast to other compounds, chrysin did not cause damage to other glial cell lines (detroit551, NIH3T3, EOC13.31 and rat mixed glial cells), suggesting that it may potentially display specific anti-glioblastoma properties without affecting normal cells [99]. The cleavage of caspase-3 and poly (ADPRibose) polymerase (PARP) was further detected upon chrysin treatment, and it was shown to reduce proliferation and induce apoptosis at high concentrations [98].

\subsection{Possible Limitations of Chrysin and Strategies to Mitigate}

Preclinical evidence supports the neuroprotective role of chrysin; however, clinical studies are limited due to the poor bioavailability of the compound $[116,117]$. The low bioavailability (less than 1\%) is mainly attributed to its poor aqueous solubility, as well as its extensive pre-systemic and first pass metabolism [118,119]. The major portion of administered chrysin remains unabsorbed and is excreted in feces, providing evidence of its poor bioavailability $[118,120-122]$. Therefore, various approaches to improving the bioavailability of chrysin should be prioritized. Chemically, the basic scaffold of chrysin could be altered to gain better bioavailability and metabolic stability, retaining its neuroprotective mechanisms. The formulation-based approaches for the enhancement of brain bioavailability appear suitable, while retaining its neuroprotective mechanisms.

In recent years, studies have been focused on the development of several formulations to improve the efficacy of chrysin by overcoming the low bioavailability issue. Nanoformulation approaches have improved brain bioavailability. Chrysin-loaded sodium oleate-based nano emulsions were shown to inhibit the first pass glucuronide conjugation of chrysin, and led to a 4-fold increase in the peak plasma concentration [119]. Chrysinloaded PLGA-PEG nanoparticle formulation enhanced the cellular uptake of chrysin in T47D and MCF7 cell lines [123]. Furthermore, co-crystals of chrysin were developed with cytosine and thiamine hydrochloride to enhance the dissolution and solubility rates by 3-4-fold, and thus, chrysin absorption was detected to be enhanced in in vivo and in vitro 
studies [124]. The development of chrysin-loaded solid lipid nanoparticles resulted in improved oral bioavailability and similar neuroprotective effects at lower doses [125]. Recently, the development of chrysin-loaded biotin-conjugated nanostructured lipid carriers (NLCs) successfully enhanced the peak plasma concentration of chrysin by 5-8-fold [126]. Overall, the nanoformulation approach has improved bioavailability and metabolic stability, while retaining the neuroprotective effect. Further, the suitability of this approach for the improvement of chrysin's bioavailability is yet to be established in a clinical setup.

\subsection{Conclusions and Future Perspectives}

Emerging pre-clinical evidence has suggested that flavonoids present a promising backbone for future drug development related to the management of neurological diseases. Chrysin has emerged as an effective flavonoid and has gained extensive research attention. The neuroprotective effect of chrysin has been demonstrated through its antioxidant, anti-inflammatory, anti-apoptotic, and MAO inhibitory potential. Despite the several pre-clinical studies highlighting the plausible role of chrysin in various neurological disorders, clinical evidence is currently lacking, mainly due to its poor bioavailability and metabolic stability. The development of synthetic analogues of chrysin and nanoformulations may be promising strategies to overcome the pharmacokinetic challenges associated with chrysin. The further development of specific brain-targeting nanoformulations and the intranasal delivery of chrysin may have additional advantages in improving brain bioavailability, bypassing the first pass effect, and building the foundations for future clinical investigations.

Author Contributions: Conceptualization, A.M. and E.A.; methodology, R.B., P.S.M., A.M.; formal analysis, A.M., N.K.; investigation, R.B., A.M.; resources, R.B., P.S.M.; data curation, A.M., R.B.; writing-original draft preparation, A.M., R.B., P.S.M.; writing-review and editing, C.P., Y.N.P., E.A.; visualization, A.M.; supervision, A.M., E.A., C.P. All authors have read and agreed to the published version of the manuscript.

Funding: This work received no external funding.

Institutional Review Board Statement: Not Applicable.

Informed Consent Statement: Not Applicable.

Data Availability Statement: No new data were created or analyzed in this study. Data sharing is not applicable to this article.

Acknowledgments: The author (AM) would like to acknowledge the Department of Pharmaceuticals, Ministry of Chemical and Fertilizers, Govt. of India, and National Institute of Pharmaceutical Education and Research (NIPER), Guwahati, for the necessary infrastructure and facilities. The images were "Created with BioRender.com".

Conflicts of Interest: The authors declare that there is no conflict of interest related to this work.

$\begin{array}{ll}\text { Abbreviations } \\ \text { 6-OHDA } & \text { 6-hydroxydopamine } \\ \text { AD } & \text { Alzheimer's diseases } \\ \text { ARE } & \text { Antioxidant response element } \\ \text { BBB } & \text { Blood-brain barrier } \\ \text { DA } & \text { Dopaminergic } \\ \text { DAMPs } & \text { Damage-associated molecular patterns } \\ \text { EAE } & \text { Experimental autoimmune encephalomyelitis } \\ \text { GPx: } & \text { Glutathione peroxidase } \\ \text { LPS } & \text { lipopolysaccharide } \\ \text { MPTP } & \text { 1-methyl-4-phenyl-1: 2, 3, 6-tetrahydropyridine; } \\ \text { MS } & \text { Multiple sclerosis } \\ \text { NF- } \text { - B } & \text { Nuclear factor kappa light chain-enhancer of activated B cells } \\ \text { Nrf2 } & \text { Nuclear factor erythroid }\end{array}$




$\begin{array}{ll}\text { PAMPs } & \text { Pathogen-associated molecular patterns } \\ \text { PD } & \text { Parkinson's disease } \\ \text { PRRs } & \text { Pattern recognition receptors } \\ \text { PTZ } & \text { Pentylenetetrazol } \\ \text { ROS } & \text { Reactive oxygen species } \\ \text { SOD } & \text { Superoxide dismutase } \\ \text { TBI } & \text { Traumatic brain injury } \\ \text { TH } & \text { Tyrosine hydroxylase } \\ \text { TLR } & \text { Toll-like receptors }\end{array}$

\section{References}

1. India State-Level Disease Burden Initiative Neurological Disorders Collaborators. The burden of neurological disorders across the states of India: The Global Burden of Disease Study 1990-2019. Lancet Glob. Health 2021, 9, e1129-e1144. [CrossRef]

2. Murray, C.J.; Vos, T.; Lozano, R.; Naghavi, M.; Flaxman, A.D.; Michaud, C.; Ezzati, M.; Shibuya, K.; Salomon, J.A.; Abdalla, S.; et al. Disability-adjusted life years (DALYs) for 291 diseases and injuries in 21 regions, 1990-2010: A systematic analysis for the Global Burden of Disease Study 2010. Lancet 2012, 380, 2197-2223. [CrossRef]

3. Maan, G.; Sikdar, B.; Kumar, A.; Shukla, R.; Mishra, A. Role of Flavonoids in Neurodegenerative Diseases: Limitations and Future Perspectives. Curr. Top. Med. Chem. 2020, 20, 1169-1194. [CrossRef]

4. Mishra, A.; Bandopadhyay, R.; Singh, P.K.; Mishra, P.S.; Sharma, N.; Khurana, N. Neuroinflammation in neurological disorders: Pharmacotherapeutic targets from bench to bedside. Metab. Brain Dis. 2021, 36, 1591-1626. [CrossRef]

5. Angelopoulou, E.; Paudel, Y.N.; Piperi, C.; Mishra, A. Neuroprotective potential of cinnamon and its metabolites in Parkinson's disease: Mechanistic insights, limitations, and novel therapeutic opportunities. J. Biochem. Mol. Toxicol. 2021, 35, e22720. [CrossRef]

6. Mani, R.; Natesan, V. Chrysin: Sources, beneficial pharmacological activities, and molecular mechanism of action. Phytochemistry 2018, 145, 187-196. [CrossRef]

7. Ayaz, M.; Sadiq, A.; Junaid, M.; Ullah, F.; Ovais, M.; Ullah, I.; Ahmed, J.; Shahid, M. Flavonoids as Prospective Neuroprotectants and Their Therapeutic Propensity in Aging Associated Neurological Disorders. Front. Aging Neurosci. 2019, 11, 155. [CrossRef]

8. Choi, Y.T.; Jung, C.H.; Lee, S.R.; Bae, J.H.; Baek, W.K.; Suh, M.H.; Park, J.; Park, C.W.; Suh, S.I. The green tea polyphenol (-)-epigallocatechin gallate attenuates beta-amyloid-induced neurotoxicity in cultured hippocampal neurons. Life Sci. 2001, 70, 603-614. [CrossRef]

9. Shukitt-Hale, B.; Cheng, V.; Joseph, J.A. Effects of blackberries on motor and cognitive function in aged rats. Nutr Neurosci. 2009, 12, 135-140. [CrossRef] [PubMed]

10. Manach, C.; Scalbert, A.; Morand, C.; Remesy, C.; Jimenez, L. Polyphenols: Food sources and bioavailability. Am. J. Clin. Nutr 2004, 79, 727-747. [CrossRef]

11. Spencer, J.P.; Abd-el-Mohsen, M.M.; Rice-Evans, C. Cellular uptake and metabolism of flavonoids and their metabolites: Implications for their bioactivity. Arch. Biochem Biophys 2004, 423, 148-161. [CrossRef]

12. Yao, W.; Cheng, J.; Kandhare, A.D.; Mukherjee-Kandhare, A.A.; Bodhankar, S.L.; Lu, G. Toxicological evaluation of a flavonoid, chrysin: Morphological, behavioral, biochemical and histopathological assessments in rats. Drug Chem. Toxicol. 2021, 44, 601-612. [CrossRef] [PubMed]

13. Walle, T.; Otake, Y.; Brubaker, J.A.; Walle, U.K.; Halushka, P.V. Disposition and metabolism of the flavonoid chrysin in normal volunteers. Br. J. Clin. Pharmacol. 2001, 51, 143-146. [PubMed]

14. Chen, Y.H.; Yang, Z.S.; Wen, C.C.; Chang, Y.S.; Wang, B.C.; Hsiao, C.A.; Shih, T.L. Evaluation of the structure-activity relationship of flavonoids as antioxidants and toxicants of zebrafish larvae. Food Chem 2012, 134, 717-724. [CrossRef]

15. Zhang, Y.; Zhao, J.; Afzal, O.; Kazmi, I.; Al-Abbasi, F.A.; Altamimi, A.S.A.; Yang, Z. Neuroprotective role of chrysin-loaded poly(lactic-co-glycolic acid) nanoparticle against kindling-induced epilepsy through Nrf2/ARE/HO-1 pathway. J. Biochem Mol. Toxicol 2021, 35, e22634. [CrossRef]

16. Skulachev, V.P. Membrane-linked systems preventing superoxide formation. Biosci Rep. 1997, 17, 347-366. [CrossRef]

17. Lin, M.T.; Beal, M.F. Mitochondrial dysfunction and oxidative stress in neurodegenerative diseases. Nature 2006, 443, 787-795. [CrossRef] [PubMed]

18. Moldogazieva, N.T.; Lutsenko, S.V.; Terentiev, A.A. Reactive Oxygen and Nitrogen Species-Induced Protein Modifications: Implication in Carcinogenesis and Anticancer Therapy. Cancer Res. 2018, 78, 6040-6047. [CrossRef]

19. Belli, S.; Rossi, M.; Molasky, N.; Middleton, L.; Caldwell, C.; Bartow-McKenney, C.; Duong, M.; Chiu, J.; Gibbs, E.; Caldwell, A.; et al. Effective and Novel Application of Hydrodynamic Voltammetry to the Study of Superoxide Radical Scavenging by Natural Phenolic Antioxidants. Antioxidants 2019, 8, 14. [CrossRef]

20. Khezri, S.; Sabzalipour, T.; Jahedsani, A.; Azizian, S.; Atashbar, S.; Salimi, A. Chrysin ameliorates aluminum phosphide-induced oxidative stress and mitochondrial damages in rat cardiomyocytes and isolated mitochondria. Environ. Toxicol 2020, 35, 1114-1124. [CrossRef]

21. El-Sisi, A.E.; El-Sayad, M.E.; Abdelsalam, N.M. Protective effects of mirtazapine and chrysin on experimentally induced testicular damage in rats. Biomed. Pharmacother. 2017, 95, 1059-1066. [CrossRef] 
22. Vedagiri, A.; Thangarajan, S. Mitigating effect of chrysin loaded solid lipid nanoparticles against Amyloid $\beta_{25-35}$ induced oxidative stress in rat hippocampal region: An efficient formulation approach for Alzheimer's disease. Neuropeptides 2016, 58, 111-125. [CrossRef]

23. Zhu, Z.Y.; Chen, L.; Liu, F.; Chen, L.J.; Meng, M.; Sun, H.Q.; Zhang, Y.M. Preparation and activity evaluation of chrysin-beta-Dgalactopyranoside. Arch. Pharm. Res. 2016, 39, 1433-1440. [CrossRef] [PubMed]

24. Chovanova, K.; Kamlarova, A.; Maresch, D.; Harichova, J.; Zamocky, M. Expression of extracellular peroxidases and catalases in mesophilic and thermophilic Chaetomia in response to environmental oxidative stress stimuli. Ecotoxicol. Environ. Saf. 2019, 181, 481-490. [CrossRef] [PubMed]

25. Wang, Y.; Branicky, R.; Noe, A.; Hekimi, S. Superoxide dismutases: Dual roles in controlling ROS damage and regulating ROS signaling. J. Cell Biol. 2018, 217, 1915-1928. [CrossRef] [PubMed]

26. Souza, L.C.; Antunes, M.S.; Filho, C.B.; Del Fabbro, L.; de Gomes, M.G.; Goes, A.T.; Donato, F.; Prigol, M.; Boeira, S.P.; Jesse, C.R. Flavonoid Chrysin prevents age-related cognitive decline via attenuation of oxidative stress and modulation of BDNF levels in aged mouse brain. Pharmacol. Biochem. Behav. 2015, 134, 22-30. [CrossRef]

27. Feng, Y.C.; Liao, C.Y.; Xia, W.K.; Jiang, X.Z.; Shang, F.; Yuan, G.R.; Wang, J.J. Regulation of three isoforms of SOD gene by environmental stresses in citrus red mite, Panonychus citri. Exp. Appl. Acarol. 2015, 67, 49-63. [CrossRef]

28. Blokhina, O.; Virolainen, E.; Fagerstedt, K.V. Antioxidants, oxidative damage and oxygen deprivation stress: A review. Ann. Bot. 2003, 91, 179-194. [CrossRef]

29. Ayala, A.; Munoz, M.F.; Arguelles, S. Lipid peroxidation: Production, metabolism, and signaling mechanisms of malondialdehyde and 4-hydroxy-2-nonenal. Oxid Med. Cell Longev. 2014, 2014, 360438. [CrossRef]

30. Feussner, I.; Wasternack, C. The lipoxygenase pathway. Annu. Rev. Plant. Biol. 2002, 53, 275-297. [CrossRef] [PubMed]

31. Jones, D.P. Redefining oxidative stress. Antioxid Redox Signal. 2006, 8, 1865-1879. [CrossRef] [PubMed]

32. Ileriturk, M.; Benzer, F.; Aksu, E.H.; Yildirim, S.; Kandemir, F.M.; Dogan, T.; Dortbudak, M.B.; Genc, A. Chrysin protects against testicular toxicity caused by lead acetate in rats with its antioxidant, anti-inflammatory, and antiapoptotic properties. J. Food Biochem. 2021, 45, e13593. [CrossRef] [PubMed]

33. Carson, M.J.; Doose, J.M.; Melchior, B.; Schmid, C.D.; Ploix, C.C. CNS immune privilege: Hiding in plain sight. Immunol. Rev. 2006, 213, 48-65. [CrossRef] [PubMed]

34. Carson, M.J.; Thrash, J.C.; Walter, B. The cellular response in neuroinflammation: The role of leukocytes, microglia and astrocytes in neuronal death and survival. Clin. Neurosci. Res. 2006, 6, 237-245. [CrossRef]

35. Ha, S.K.; Moon, E.; Kim, S.Y. Chrysin suppresses LPS-stimulated proinflammatory responses by blocking NF-kappaB and JNK activations in microglia cells. Neurosci. Lett 2010, 485, 143-147. [CrossRef]

36. Lyman, M.; Lloyd, D.G.; Ji, X.; Vizcaychipi, M.P.; Ma, D. Neuroinflammation: The role and consequences. Neurosci Res. 2014, 79, 1-12. [CrossRef]

37. Park, S.E.; Sapkota, K.; Kim, S.; Kim, H.; Kim, S.J. Kaempferol acts through mitogen-activated protein kinases and protein kinase B/AKT to elicit protection in a model of neuroinflammation in BV2 microglial cells. Br. J. Pharmacol. 2011, 164, 1008-1025. [CrossRef]

38. Glass, C.K.; Saijo, K.; Winner, B.; Marchetto, M.C.; Gage, F.H. Mechanisms underlying inflammation in neurodegeneration. Cell 2010, 140, 918-934. [CrossRef]

39. Harry, G.J.; Kraft, A.D. Neuroinflammation and microglia: Considerations and approaches for neurotoxicity assessment. Expert Opin. Drug. Metab. Toxicol 2008, 4, 1265-1277. [CrossRef]

40. Kaushal, V.; Schlichter, L.C. Mechanisms of microglia-mediated neurotoxicity in a new model of the stroke penumbra. J. Neurosci. 2008, 28, 2221-2230. [CrossRef]

41. Walsh, J.G.; Muruve, D.A.; Power, C. Inflammasomes in the CNS. Nat. Rev. Neurosci. 2014, 15, 84-97. [CrossRef] [PubMed]

42. Shi, J.; Gao, W.; Shao, F. Pyroptosis: Gasdermin-Mediated Programmed Necrotic Cell Death. Trends Biochem. Sci. 2017, 42, 245-254. [CrossRef] [PubMed]

43. Lamkanfi, M.; Dixit, V.M. Mechanisms and functions of inflammasomes. Cell 2014, 157, 1013-1022. [CrossRef] [PubMed]

44. Khombi Shooshtari, M.; Farbood, Y.; Mansouri, S.M.T.; Badavi, M.; Khorsandi, L.S.; Ghasemi Dehcheshmeh, M.; Sarkaki, A.R. Neuroprotective Effects of Chrysin Mediated by Estrogenic Receptors Following Cerebral Ischemia and Reperfusion in Male Rats. Basic Clin. Neurosci. 2021, 12, 149-162. [CrossRef]

45. Li, T.F.; Ma, J.; Han, X.W.; Jia, Y.X.; Yuan, H.F.; Shui, S.F.; Guo, D.; Yan, L. Chrysin ameliorates cerebral ischemia/reperfusion (I/R) injury in rats by regulating the PI3K/Akt/mTOR pathway. Neurochem. Int. 2019, 129, 104496. [CrossRef] [PubMed]

46. Lee, B.K.; Lee, W.J.; Jung, Y.S. Chrysin Attenuates VCAM-1 Expression and Monocyte Adhesion in Lipopolysaccharide-Stimulated Brain Endothelial Cells by Preventing NF-kappaB Signaling. Int. J. Mol. Sci. 2017, 18, 1424. [CrossRef]

47. Mantawy, E.M.; El-Bakly, W.M.; Esmat, A.; Badr, A.M.; El-Demerdash, E. Chrysin alleviates acute doxorubicin cardiotoxicity in rats via suppression of oxidative stress, inflammation and apoptosis. Eur. J. Pharmacol. 2014, 728, 107-118. [CrossRef]

48. Chang, Y.H.; Chiang, Y.F.; Chen, H.Y.; Huang, Y.J.; Wang, K.L.; Hong, Y.H.; Ali, M.; Shieh, T.M.; Hsia, S.M. Anti-Inflammatory and Anti-Hyperuricemic Effects of Chrysin on a High Fructose Corn Syrup-Induced Hyperuricemia Rat Model via the Amelioration of Urate Transporters and Inhibition of NLRP3 Inflammasome Signaling Pathway. Antioxidants 2021, 10, 564. [CrossRef]

49. Liao, T.; Ding, L.; Wu, P.; Zhang, L.; Li, X.; Xu, B.; Zhang, H.; Ma, Z.; Xiao, Y.; Wang, P. Chrysin Attenuates the NLRP3 Inflammasome Cascade to Reduce Synovitis and Pain in KOA Rats. Drug Des. Devel. Ther. 2020, 14, 3015-3027. [CrossRef] 
50. Wang, L.; Kou, M.C.; Weng, C.Y.; Hu, L.W.; Wang, Y.J.; Wu, M.J. Arsenic modulates heme oxygenase-1, interleukin-6, and vascular endothelial growth factor expression in endothelial cells: Roles of ROS, NF-kappaB, and MAPK pathways. Arch. Toxicol. 2012, 86, 879-896. [CrossRef]

51. Qi, S.M.; Li, Q.; Jiang, Q.; Qi, Z.L.; Zhang, Y. Chrysin inhibits lipopolysaccharide-induced inflammatory responses of macrophages via JAK-STATs signaling pathway. Nan Fang Yi Ke Da Xue Xue Bao 2018, 38, 243-250. [PubMed]

52. Elmore, S. Apoptosis: A review of programmed cell death. Toxicol. Pathol. 2007, 35, 495-516. [CrossRef] [PubMed]

53. Wajant, H. The Fas signaling pathway: More than a paradigm. Science 2002, 296, 1635-1636. [CrossRef] [PubMed]

54. Scaffidi, C.; Fulda, S.; Srinivasan, A.; Friesen, C.; Li, F.; Tomaselli, K.J.; Debatin, K.M.; Krammer, P.H.; Peter, M.E. Two CD95 (APO-1/Fas) signaling pathways. EMBO J. 1998, 17, 1675-1687. [CrossRef] [PubMed]

55. Hsu, H.; Xiong, J.; Goeddel, D.V. The TNF receptor 1-associated protein TRADD signals cell death and NF-kappa B activation. Cell 1995, 81, 495-504. [CrossRef]

56. Saelens, X.; Festjens, N.; Vande Walle, L.; van Gurp, M.; van Loo, G.; Vandenabeele, P. Toxic proteins released from mitochondria in cell death. Oncogene 2004, 23, 2861-2874. [CrossRef]

57. Du, C.; Fang, M.; Li, Y.; Li, L.; Wang, X. Smac, a mitochondrial protein that promotes cytochrome c-dependent caspase activation by eliminating IAP inhibition. Cell 2000, 102, 33-42. [CrossRef]

58. Schimmer, A.D. Inhibitor of apoptosis proteins: Translating basic knowledge into clinical practice. Cancer Res. 2004, 64, 7183-7190. [CrossRef]

59. Ayna, A.; Ozbolat, S.N.; Darendelioglu, E. Quercetin, chrysin, caffeic acid and ferulic acid ameliorate cyclophosphamide-induced toxicities in SH-SY5Y cells. Mol. Biol. Rep. 2020, 47, 8535-8543. [CrossRef]

60. Darendelioglu, E. Neuroprotective Effects of Chrysin on Diclofenac-Induced Apoptosis in SH-SY5Y Cells. Neurochem Res. 2020, 45, 1064-1071. [CrossRef]

61. Guo, B.; Zheng, C.; Cai, W.; Cheng, J.; Wang, H.; Li, H.; Sun, Y.; Cui, W.; Wang, Y.; Han, Y.; et al. Multifunction of Chrysin in Parkinson's Model: Anti-Neuronal Apoptosis, Neuroprotection via Activation of MEF2D, and Inhibition of Monoamine Oxidase-B. J. Agric. Food Chem. 2016, 64, 5324-5333. [CrossRef] [PubMed]

62. Rashno, M.; Sarkaki, A.; Farbood, Y.; Rashno, M.; Khorsandi, L.; Naseri, M.K.G.; Dianat, M. Therapeutic effects of chrysin in a rat model of traumatic brain injury: A behavioral, biochemical, and histological study. Life Sci. 2019, 228, 285-294. [CrossRef]

63. Thangarajan, S.; Ramachandran, S.; Krishnamurthy, P. Chrysin exerts neuroprotective effects against 3-Nitropropionic acid induced behavioral despair-Mitochondrial dysfunction and striatal apoptosis via upregulating Bcl-2 gene and downregulating Bax-Bad genes in male wistar rats. Biomed. Pharmacother. 2016, 84, 514-525. [CrossRef] [PubMed]

64. Meiser, J.; Weindl, D.; Hiller, K. Complexity of dopamine metabolism. Cell Commun. Signal. 2013, 11, 34. [CrossRef]

65. Del Fabbro, L.; Rossito Goes, A.; Jesse, C.R.; de Gomes, M.G.; Cattelan Souza, L.; Lobo Ladd, F.V.; Lobo Ladd, A.A.B.; Nunes Arantes, R.V.; Reis Simionato, A.; Oliveira, M.S.; et al. Chrysin protects against behavioral, cognitive and neurochemical alterations in a 6-hydroxydopamine model of Parkinson's disease. Neurosci. Lett. 2019, 706, 158-163. [CrossRef] [PubMed]

66. Bortolotto, V.C.; Pinheiro, F.C.; Araujo, S.M.; Poetini, M.R.; Bertolazi, B.S.; de Paula, M.T.; Meichtry, L.B.; de Almeida, F.P.; de Freitas Couto, S.; Jesse, C.R.; et al. Chrysin reverses the depressive-like behavior induced by hypothyroidism in female mice by regulating hippocampal serotonin and dopamine. Eur. J. Pharmacol. 2018, 822, 78-84. [CrossRef]

67. Larit, F.; Elokely, K.M.; Chaurasiya, N.D.; Benyahia, S.; Nael, M.A.; Leon, F.; Abu-Darwish, M.S.; Efferth, T.; Wang, Y.H.; Belouahem-Abed, D.; et al. Inhibition of human monoamine oxidase A and B by flavonoids isolated from two Algerian medicinal plants. Phytomedicine 2018, 40, 27-36. [CrossRef]

68. Krishnamoorthy, A.; Sevanan, M.; Mani, S.; Balu, M.; Balaji, S.; Ramajayan, P. Chrysin restores MPTP induced neuroinflammation, oxidative stress and neurotrophic factors in an acute Parkinson's disease mouse model. Neurosci. Lett. 2019, $709,134382$. [CrossRef]

69. Ngo, D.H.; Vo, T.S. An Updated Review on Pharmaceutical Properties of Gamma-Aminobutyric Acid. Molecules 2019, 24, 2678. [CrossRef]

70. Nuss, P. Anxiety disorders and GABA neurotransmission: A disturbance of modulation. Neuropsychiatr. Dis. Treat. 2015, 11, 165-175.

71. Marder, M.; Paladini, A.C. GABA(A)-receptor ligands of flavonoid structure. Curr. Top. Med. Chem. 2002, 2, 853-867. [CrossRef] [PubMed]

72. Rodriguez-Landa, J.F.; Guillen-Ruiz, G.; Hernandez-Lopez, F.; Cueto-Escobedo, J.; Rivadeneyra-Dominguez, E.; Bernal-Morales, B.; Herrera-Huerta, E.V. Chrysin reduces anxiety-like behavior through actions on GABAA receptors during metestrus-diestrus in the rat. Behav. Brain Res. 2021, 397, 112952. [CrossRef] [PubMed]

73. Cueto-Escobedo, J.; Andrade-Soto, J.; Lima-Maximino, M.; Maximino, C.; Hernandez-Lopez, F.; Rodriguez-Landa, J.F. Involvement of GABAergic system in the antidepressant-like effects of chrysin (5,7-dihydroxyflavone) in ovariectomized rats in the forced swim test: Comparison with neurosteroids. Behav. Brain Res. 2020, 386, 112590. [CrossRef] [PubMed]

74. Cho, I.; Song, H.O.; Cho, J.H. Flavonoids mitigate neurodegeneration in aged Caenorhabditis elegans by mitochondrial uncoupling. Food Sci. Nutr. 2020, 8, 6633-6642. [CrossRef]

75. Cho, I.; Song, H.O.; Cho, J.H. Mitochondrial Uncoupling Attenuates Age-Dependent Neurodegeneration in C. elegans. Mol. Cells 2017, 40, 864-870. 
76. Ahmed, M.R.; Shaikh, M.A.; Ul Haq, S.H.I.; Nazir, S. Neuroprotective role of chrysin in attenuating loss of dopaminergic neurons and improving motor, learning and memory functions in rats. Int. J. Health Sci. 2018, 12, 35-43.

77. Goes, A.T.R.; Jesse, C.R.; Antunes, M.S.; Lobo Ladd, F.V.; Lobo Ladd, A.A.B.; Luchese, C.; Paroul, N.; Boeira, S.P. Protective role of chrysin on 6-hydroxydopamine-induced neurodegeneration a mouse model of Parkinson's disease: Involvement of neuroinflammation and neurotrophins. Chem. Biol. Interact. 2018, 279, 111-120. [CrossRef] [PubMed]

78. Zhang, K.; Ge, Z.; Xue, Z.; Huang, W.; Mei, M.; Zhang, Q.; Li, Y.; Li, W.; Zhang, Z.; Zhang, Z.; et al. Chrysin suppresses human CD14(+) monocyte-derived dendritic cells and ameliorates experimental autoimmune encephalomyelitis. J. Neuroimmunol. 2015, 288, 13-20. [CrossRef]

79. Mercer, L.D.; Kelly, B.L.; Horne, M.K.; Beart, P.M. Dietary polyphenols protect dopamine neurons from oxidative insults and apoptosis: Investigations in primary rat mesencephalic cultures. Biochem. Pharmacol. 2005, 69, 339-345. [CrossRef]

80. Alkahtane, A.A.; Alghamdi, H.A.; Almutairi, B.; Khan, M.M.; Hasnain, M.S.; Abdel-Daim, M.M.; Alghamdi, W.M.; Alkahtani, S. Inhibition of human amylin aggregation by Flavonoid Chrysin: An in-silico and in-vitro approach. Int. J. Med. Sci. 2021, 18, 199-206. [CrossRef]

81. Hirsch, E.C.; Standaert, D.G. Ten Unsolved Questions about Neuroinflammation in Parkinson's Disease. Mov. Disord. 2021, 36, 16-24. [CrossRef]

82. Giacomeli, R.; de Gomes, M.G.; Reolon, J.B.; Haas, S.E.; Colome, L.M.; Jesse, C.R. Chrysin loaded lipid-core nanocapsules ameliorates neurobehavioral alterations induced by beta-amyloid1-42 in aged female mice. Behav. Brain Res. 2020, $390,112696$. [CrossRef] [PubMed]

83. Nday, C.M.; Eleftheriadou, D.; Jackson, G. Magnetic chrysin silica nanomaterials behavior in an amyloidogenic environment. Hell. J. Nucl. Med. 2019, 22, 42-50.

84. Mansour, S.Z.; Moawed, F.S.M.; Elmarkaby, S.M. Protective effect of 5, 7-dihydroxyflavone on brain of rats exposed to acrylamide or gamma-radiation. J. Photochem. Photobiol. B 2017, 175, 149-155. [CrossRef]

85. Sharma, P.; Kumari, A.; Gulati, A.; Krishnamurthy, S.; Hemalatha, S. Chrysin isolated from Pyrus pashia fruit ameliorates convulsions in experimental animals. Nutr. Neurosci. 2019, 22, 569-577. [CrossRef]

86. Singh, B.; Singh, D.; Goel, R.K. Dual protective effect of Passiflora incarnata in epilepsy and associated post-ictal depression. J. Ethnopharmacol. 2012, 139, 273-279. [CrossRef]

87. Singh, B.; Mishra, A.; Goel, R.K. Anticonvulsant activity of Passiflora incarnata: No role of chrysin. J. Pharm. Negat. Results 2011, 2, $51-54$.

88. Medina, J.H.; Paladini, A.C.; Wolfman, C.; Levi de Stein, M.; Calvo, D.; Diaz, L.E.; Pena, C. Chrysin (5,7-di-OH-flavone), a naturally-occurring ligand for benzodiazepine receptors, with anticonvulsant properties. Biochem. Pharmacol. 1990, 40, 2227-2231. [CrossRef]

89. Rashno, M.; Ghaderi, S.; Nesari, A.; Khorsandi, L.; Farbood, Y.; Sarkaki, A. Chrysin attenuates traumatic brain injury-induced recognition memory decline, and anxiety/depression-like behaviors in rats: Insights into underlying mechanisms. Psychopharmacology 2020, 237, 1607-1619. [CrossRef]

90. Shooshtari, M.K.; Sarkaki, A.; Mansouri, S.M.T.; Badavi, M.; Khorsandi, L.; Ghasemi Dehcheshmeh, M.; Farbood, Y. Protective effects of Chrysin against memory impairment, cerebral hyperemia and oxidative stress after cerebral hypoperfusion and reperfusion in rats. Metab. Brain Dis. 2020, 35, 401-412. [CrossRef] [PubMed]

91. Sarkaki, A.; Farbood, Y.; Mansouri, S.M.T.; Badavi, M.; Khorsandi, L.; Dehcheshmeh, M.G.; Shooshtari, M.K. Chrysin prevents cognitive and hippocampal long-term potentiation deficits and inflammation in rat with cerebral hypoperfusion and reperfusion injury. Life Sci. 2019, 226, 202-209. [CrossRef]

92. El Khashab, I.H.; Abdelsalam, R.M.; Elbrairy, A.I.; Attia, A.S. Chrysin attenuates global cerebral ischemic reperfusion injury via suppression of oxidative stress, inflammation and apoptosis. Biomed. Pharmacother. 2019, 112, 108619. [CrossRef]

93. Durak, M.A.; Oztanir, M.N.; Basak Turkmen, N.; Ciftci, O.; Taslidere, A.; Tecellioglu, M.; Onder, A. Chrysin prevents brain damage caused by global cerebralischemia/reperfusion in a C57BL/J6 mouse model. Turk. J. Med. Sci. 2016, 46, 1926-1933. [CrossRef] [PubMed]

94. Yao, Y.; Chen, L.; Xiao, J.; Wang, C.; Jiang, W.; Zhang, R.; Hao, J. Chrysin protects against focal cerebral ischemia/reperfusion injury in mice through attenuation of oxidative stress and inflammation. Int. J. Mol. Sci. 2014, 15, 20913-20926. [CrossRef] [PubMed]

95. He, X.L.; Wang, Y.H.; Bi, M.G.; Du, G.H. Chrysin improves cognitive deficits and brain damage induced by chronic cerebral hypoperfusion in rats. Eur.. Pharmacol. 2012, 680, 41-48. [CrossRef]

96. Wang, J.; Wang, H.; Sun, K.; Wang, X.; Pan, H.; Zhu, J.; Ji, X.; Li, X. Chrysin suppresses proliferation, migration, and invasion in glioblastoma cell lines via mediating the ERK/Nrf2 signaling pathway. Drug Des. Devel. Ther. 2018, 12, 721-733. [CrossRef]

97. Jia, W.Z.; Zhao, J.C.; Sun, X.L.; Yao, Z.G.; Wu, H.L.; Xi, Z.Q. Additive anticancer effects of chrysin and low dose cisplatin in human malignant glioma cell (U87) proliferation and evaluation of the mechanistic pathway. J. BUON 2015, 20, 1327-1336.

98. Santos, B.L.; Oliveira, M.N.; Coelho, P.L.; Pitanga, B.P.; da Silva, A.B.; Adelita, T.; Silva, V.D.; Costa Mde, F.; El-Bacha, R.S.; Tardy, M.; et al. Flavonoids suppress human glioblastoma cell growth by inhibiting cell metabolism, migration, and by regulating extracellular matrix proteins and metalloproteinases expression. Chem. Biol. Interact. 2015, 242, 123-138. [CrossRef] [PubMed] 
99. Liao, C.L.; Chen, C.M.; Chang, Y.Z.; Liu, G.Y.; Hung, H.C.; Hsieh, T.Y.; Lin, C.L. Pine (Pinus morrisonicola Hayata) needle extracts sensitize GBM8901 human glioblastoma cells to temozolomide by downregulating autophagy and O(6)-methylguanine-DNA methyltransferase expression. J. Agric. Food Chem. 2014, 62, 10458-10467. [CrossRef] [PubMed]

100. Weng, M.S.; Ho, Y.S.; Lin, J.K. Chrysin induces G1 phase cell cycle arrest in C6 glioma cells through inducing p21Waf1/Cip1 expression: Involvement of p38 mitogen-activated protein kinase. Biochem. Pharmacol. 2005, 69, 1815-1827. [CrossRef]

101. Del Fabbro, L.; de Gomes, M.G.; Souza, L.C.; Goes, A.R.; Boeira, S.P.; Oliveira, M.S.; Furian, A.F.; Jesse, C.R. Chrysin suppress immune responses and protects from experimental autoimmune encephalomyelitis in mice. J. Neuroimmunol. 2019, $335,577007$. [CrossRef]

102. Zhang, Z.; Li, G.; Szeto, S.S.W.; Chong, C.M.; Quan, Q.; Huang, C.; Cui, W.; Guo, B.; Wang, Y.; Han, Y.; et al. Examining the neuroprotective effects of protocatechuic acid and chrysin on in vitro and in vivo models of Parkinson disease. Free Radic. Biol. Med. 2015, 84, 331-343. [CrossRef]

103. Li, Z.; Chu, S.; He, W.; Zhang, Z.; Liu, J.; Cui, L.; Yan, X.; Li, D.; Chen, N. A20 as a novel target for the anti-neuroinflammatory effect of chrysin via inhibition of NF-kappaB signaling pathway. Brain Behav. Immun. 2019, 79, 228-235. [CrossRef]

104. Mani, R.; Natesan, V.; Arumugam, R. Neuroprotective effect of chrysin on hyperammonemia mediated neuroinflammatory responses and altered expression of astrocytic protein in the hippocampus. Biomed. Pharmacother. 2017, 88, 762-769. [CrossRef]

105. Filho, C.B.; Jesse, C.R.; Donato, F.; Giacomeli, R.; Del Fabbro, L.; da Silva Antunes, M.; de Gomes, M.G.; Goes, A.T.; Boeira, S.P.; Prigol, M.; et al. Chronic unpredictable mild stress decreases BDNF and NGF levels and $\mathrm{Na}(+), \mathrm{K}(+)$-ATPase activity in the hippocampus and prefrontal cortex of mice: Antidepressant effect of chrysin. Neuroscience 2015, 289, 367-380. [CrossRef]

106. Jiang, Y.; Gong, F.L.; Zhao, G.B.; Li, J. Chrysin suppressed inflammatory responses and the inducible nitric oxide synthase pathway after spinal cord injury in rats. Int. J. Mol. Sci. 2014, 15, 12270-12279. [CrossRef]

107. Mehri, S.; Karami, H.V.; Hassani, F.V.; Hosseinzadeh, H. Chrysin reduced acrylamide-induced neurotoxicity in both in vitro and in vivo assessments. Iran. Biomed. J. 2014, 18, 101-106.

108. Cummings, J. New approaches to symptomatic treatments for Alzheimer's disease. Mol. Neurodegener. 2021, 16, 2. [CrossRef]

109. Qi, Y.; Cheng, X.; Jing, H.; Yan, T.; Xiao, F.; Wu, B.; Bi, K.; Jia, Y. Comparative pharmacokinetic study of the components in Alpinia oxyphylla Miq.-Schisandra chinensis (Turcz.) Baill. herb pair and its single herb between normal and Alzheimer's disease rats by UPLC-MS/MS. J. Pharm. Biomed. Anal. 2020, 177, 112874. [CrossRef]

110. Bortolotto, V.C.; Araujo, S.M.; Pinheiro, F.C.; Poetini, M.R.; de Paula, M.T.; Meichtry, L.B.; de Almeida, F.P.; Musachio, E.A.S.; Guerra, G.P.; Prigol, M. Modulation of glutamate levels and $\mathrm{Na}(+), \mathrm{K}(+)$-ATPase activity contributes to the chrysin memory recovery in hypothyroidism mice. Physiol. Behav. 2020, 222, 112892. [CrossRef]

111. Akyuz, E.; Polat, A.K.; Eroglu, E.; Kullu, I.; Angelopoulou, E.; Paudel, Y.N. Revisiting the role of neurotransmitters in epilepsy: An updated review. Life Sci. 2021, 265, 118826. [CrossRef]

112. Mishra, A.; Goel, R.K. Modulatory Effect of Serotonergic System in Pentylenetetrazole-Induced Seizures and Associated Memory Deficit: Role of 5-HT1A and 5-HT2A/2C. J. Epilepsy Res. 2019, 9, 119-125. [CrossRef] [PubMed]

113. Mishra, A.; Goel, R.K. Chronic 5-HT3 receptor antagonism ameliorates seizures and associated memory deficit in pentylenetetrazole-kindled mice. Neuroscience 2016, 339, 319-328. [CrossRef]

114. Stompor-Goracy, M.; Bajek-Bil, A.; Machaczka, M. Chrysin: Perspectives on Contemporary Status and Future Possibilities as Pro-Health Agent. Nutrients 2021, 13, 2038. [CrossRef] [PubMed]

115. Markiewicz-Zukowska, R.; Borawska, M.H.; Fiedorowicz, A.; Naliwajko, S.K.; Sawicka, D.; Car, H. Propolis changes the anticancer activity of temozolomide in U87MG human glioblastoma cell line. BMC Complement. Altern. Med. 2013, 13, 50. [CrossRef]

116. Balakrishnan, R.; Azam, S.; Cho, D.Y.; Su-Kim, I.; Choi, D.K. Natural Phytochemicals as Novel Therapeutic Strategies to Prevent and Treat Parkinson's Disease: Current Knowledge and Future Perspectives. Oxid Med. Cell Longev. 2021, $2021,6680935$.

117. Gao, S.; Siddiqui, N.; Etim, I.; Du, T.; Zhang, Y.; Liang, D. Developing nutritional component chrysin as a therapeutic agent: Bioavailability and pharmacokinetics consideration, and ADME mechanisms. Biomed. Pharmacother. 2021, 142, 112080. [CrossRef]

118. Hofer, S.J.; Davinelli, S.; Bergmann, M.; Scapagnini, G.; Madeo, F. Caloric Restriction Mimetics in Nutrition and Clinical Trials. Front. Nutr. 2021, 8, 717343. [CrossRef]

119. Dong, D.; Quan, E.; Yuan, X.; Xie, Q.; Li, Z.; Wu, B. Sodium Oleate-Based Nanoemulsion Enhances Oral Absorption of Chrysin through Inhibition of UGT-Mediated Metabolism. Mol. Pharm. 2017, 14, 2864-2874. [CrossRef]

120. Ge, S.; Gao, S.; Yin, T.; Hu, M. Determination of pharmacokinetics of chrysin and its conjugates in wild-type FVB and Bcrp1 knockout mice using a validated LC-MS/MS method. J. Agric. Food Chem. 2015, 63, 2902-2910. [CrossRef]

121. Mohos, V.; Fliszár-Nyúl, E.; Schilli, G.; Hetényi, C.; Lemli, B.; Kunsági-Máté, S.; Bognár, B.; Poór, M. Interaction of Chrysin and Its Main Conjugated Metabolites Chrysin-7-Sulfate and Chrysin-7-Glucuronide with Serum Albumin. Int. J. Mol. Sci. 2018, 19, 4073. [CrossRef]

122. Mohos, V.; Fliszár-Nyúl, E.; Ungvári, O.; Bakos, É.; Kuffa, K.; Bencsik, T.; Zsidó, B.Z.; Hetényi, C.; Telbisz, Á.; Özvegy-Laczka, C.; et al. Effects of Chrysin and Its Major Conjugated Metabolites Chrysin-7-Sulfate and Chrysin-7-Glucuronide on Cytochrome P450 Enzymes and on OATP, P-gp, BCRP, and MRP2 Transporters. Drug Metab. Dispos. 2020, 48, 1064-1073. [CrossRef]

123. Anari, E.; Akbarzadeh, A.; Zarghami, N. Chrysin-loaded PLGA-PEG nanoparticles designed for enhanced effect on the breast cancer cell line. Artif Cells Nanomed. Biotechnol. 2016, 44, 1410-1416. [CrossRef] [PubMed]

124. Chadha, R.; Bhalla, Y.; Nandan, A.; Chadha, K.; Karan, M. Chrysin cocrystals: Characterization and evaluation. J. Pharm. Biomed. 2017, 134, 361-371. [CrossRef] [PubMed] 
125. Abbas, M. Potential Role of Nanoparticles in Treating the Accumulation of Amyloid-Beta Peptide in Alzheimer's Patients. Polymers 2021, 13, 1051. [CrossRef]

126. Sharma, T.; Katare, O.P.; Jain, A.; Jain, S.; Chaudhari, D.; Borges, B.; Singh, B. QbD-Steered Development of Biotin-Conjugated Nanostructured Lipid Carriers for Oral Delivery of Chrysin: Role of Surface Modification for Improving Biopharmaceutical Performance. Colloids Surf. B Biointerfaces. 2021, 197, 111429. [CrossRef] 